\title{
Relations between Fractional Operations and Time-Frequency Distributions, and Their Applications
}

\author{
Soo-Chang Pei, Fellow, IEEE, and Jian-Jiun Ding
}

\begin{abstract}
The fractional Fourier transform (FRFT) is a useful tool for signal processing. It is the generalization of the Fourier transform. Many fractional operations, such as fractional convolution, fractional correlation, and the fractional Hilbert transform, are defined from it. In fact, the FRFT can be further generalized into the linear canonical transform (LCT), and we can also use the LCT to define several canonical operations. In this paper, we will discuss the relations between the operations described above and some important time-frequency distributions (TFDs), such as the Wigner distribution function (WDF), the ambiguity function (AF), the signal correlation function, and the spectrum correlation function. First, we systematically review the previous works in brief. Then, some new relations are derived and listed in tables. Then, we use these relations to analyze the applications of the FRFT/LCT on the fractional/canonical filter design, fractional/canonical Hilbert transform, beam shaping, and then we analyze the phase-amplitude problems of the FRFT/LCT. For phase-amplitude problems, we find, as with the original Fourier transform, that in most cases, the phase is more important than the amplitude for the FRFT/LCT. We also use the WDF to explain why fractional/canonical convolution can be used for space-variant pattern recognition.
\end{abstract}

Index Terms-Fractional convolution, fractional correlation, fractional Fourier transform, linear canonical transform.

\section{INTRODUCTION}

$\mathbf{T}$ HE WIGNER distribution function (WDF) [11]-[13] is a powerful time-frequency analysis tool. It can be used to illustrate the time-frequency properties of signals. Meanwhile, the effects of some operations, such as the Fourier transform (FT), convolution, and correlation, can also be illustrated by it. There are also some other time-frequency analysis tools related to the WDF by the FT/IFT, such as the ambiguity function (AF), the signal correlation function, and the spectrum correlation function, etc.

The fractional Fourier transform (FRFT) [1]-[5], [24] is the generalization of the FT. It can analyze the signal in between the time and frequency domains. Its relations with WDF are discussed in [3], [14]-[16], [31], [32], and [34]. Some fractional operations, such as the fractional convolution [15], [17], [18], fractional correlation [15], [19]-[21], the fractional Hilbert transform [22]-[24], and the fractional filter [15], [24]-[27] are defined from the FRFT and can extend the utilities of the original operations.

Manuscript received October 12, 1999; revised April 26, 2001. The associate editor coordinating the review of this paper and approving it for publication was Prof. Colin F. Cowan.

The authors are with the Department of Electrical Engineering, National

Taiwan University, Taipei, Taiwan, R.O.C. (e-mail: pei@cc.ee.ntu.edu.tw).

Publisher Item Identifier S 1053-587X(01)05849-4.
The FRFT has been further generalized into the linear canonical transform (LCT) [6], [7], [9] (it is also called the special affine Fourier transform [8] or the ABCD transform [10]). We can also further extend the fractional operations by using the LCT.

In this paper, we will discuss the relations between the time-frequency distributions (TFDs) and the FRFT, the LCT, and fractional/canonical operations. We also discuss the applications of these relations and use these relations to illustrate some phenomena. In Section II, we will review some previous works. They will be used in the following parts. Then, in Sections III-VI, we will discuss the following.

1) relations between the Wigner distribution function (WDF) and fractional/canonical convolution and the role of each of the parameters of the LCT (Section III-A);

2) use of the above relations to discuss the effects of using the filter designed by FRFT/LCT and the effects of the fractional/canonical Hilbert transform (Sections III-B, III-D);

3) amplitude/phase problem for the FRFT/LCT for image processing by the inspection of WDF (Section III-C);

4) relations between WDF and fractional/canonical correlation and an explanation of why the fractional/canonical correlation can be used for space-variant pattern recognition (Section IV);

5) moment properties, canonical spectrum correlation function, and some other interesting properties between WDF and FRFT/LCT and their applications (Section V);

6) relations between the ambiguity function (AF) and the the FRFT/LCT, fractional/canonical convolution, correlation, and the Hilbert transform (Sections VI-A);

7) relations between the signal/spectrum correlation function and FRFT/LCT (Section VI-B).

\section{PRELIMINARY}

\section{A. Wigner Distribution Function}

The WDF [11]-[13] of $f(t)$ is defined as

$$
\begin{aligned}
W_{f}(t, \omega)= & 1 / 2 \pi \cdot \int_{-\infty}^{\infty} f(t+\tau / 2) \cdot f^{*}(t-\tau / 2) \\
& \cdot e^{-j \omega \tau} \cdot d \tau \\
= & 1 / 2 \pi \cdot \int_{-\infty}^{\infty} F(\omega+\eta / 2) \cdot F^{*}(\omega-\eta / 2) \\
& \cdot e^{j t \eta} \cdot d \eta
\end{aligned}
$$


where $F(\omega)$ is the Fourier transform of $f(t)$ :

$$
F(\omega)=1 / \sqrt{2 \pi} \cdot \int_{-\infty}^{\infty} f(t) \cdot e^{-j \omega t} \cdot d t .
$$

Some important properties of WDF are listed in Table I [12].

There are some other time-frequency analysis tools closely related to the WDF, and we can obtain them by doing the forward and inverse Fourier transform from the WDF:

a) Signal correlation function:

$$
C_{f}(t, \tau)=1 / \sqrt{2 \pi} \cdot f(t+\tau / 2) \cdot f^{*}(t-\tau / 2) .
$$

b) Spectrum correlation function:

$$
S_{f}(\eta, \omega)=1 / \sqrt{2 \pi} \cdot F(\omega+\eta / 2) \cdot F^{*}(\omega-\eta / 2) .
$$

c) Ambiguity function $(A F)$ :

$$
A_{f}(\eta, \tau)=1 / 2 \pi \cdot \int_{-\infty}^{\infty} f(t+\tau / 2) \cdot f^{*}(t-\tau / 2) \cdot e^{-j t \eta} \cdot d t
$$

We draw the relations among the WDF, the AF, and the signal/spectrum correlation function in Fig. 1. Other properties of the WDF and other time-frequency analysis tools can be seen in [12].

\section{B. Fractional Fourier Transform, Linear Canonical Transform, and Their Related Fractional Operations}

The FRFT [1]-[5], [24] is defined as

$$
\begin{aligned}
O_{F}^{\alpha}(f(t))= & \sqrt{\frac{1-j \cot \alpha}{2 \pi}} \cdot e^{j / 2 \cdot \cot \alpha \cdot u^{2}} \cdot \int_{-\infty}^{\infty} e^{-j \cdot \csc \alpha \cdot u \cdot t} \\
& \cdot e^{j / 2 \cdot \cot \alpha \cdot t^{2}} \cdot f(t) \cdot d t
\end{aligned}
$$

When $\alpha=\pi / 2$, it becomes the original Fourier transform (FT), and when $\alpha=0$, it will be an identity operation. It transforms a function into a intermediate domain between time and frequency when $\alpha \neq N \cdot \pi / 2$, where $N$ is an integer. It satisfies the additivity property as

$$
O_{F}^{\beta}\left(O_{F}^{\alpha}(f(t))\right)=O_{F}^{\alpha}\left(O_{F}^{\beta}(f(t))\right)=O_{F}^{\alpha+\beta}(f(t)) .
$$

The FRFT is a very useful tool for signal processing and has many applications such as optical system analysis, filter design, signal synthesis, solving differential equations, phase retrieval, and pattern recognition, etc.

The FRFT has been further generalized into the LCT [6]-[10] (which is also called the special affine transform or ABCD transform). Its definition is

$$
\begin{aligned}
& O_{F}^{(a, b, c, d)}(f(t)) \\
& =\sqrt{\frac{1}{j 2 \pi b}} \cdot e^{(j / 2)(d / b) \cdot u^{2}} \cdot \int_{-\infty}^{\infty} e^{-(j / b) \cdot u \cdot t} \\
& \quad \cdot e^{(j / 2)(a / b) \cdot t^{2}} \cdot f(t) \cdot d t \quad(a d-b c=1) .
\end{aligned}
$$

\begin{tabular}{|c|c|}
\hline (1) Projection property & $|f(t)|^{2}=\mathcal{L}_{\infty}^{\infty} W_{f}(t, \omega) d w, \quad \mid F(\varpi)^{2}=\mathcal{L}_{\infty}^{\infty} W_{f}(t, \varpi) d t$ \\
\hline (2) Recovery property & 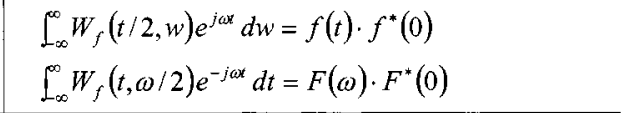 \\
\hline $\begin{array}{l}\text { (3) Mean condition } \\
\text { frequency and mean } \\
\text { condition time }\end{array}$ & $\begin{array}{l}\text { If } f(t)=|f(t)| \cdot e^{j \phi(t)}, \quad F(\omega)=|F(\omega)| \cdot e^{j \Psi(\omega)} \\
\text { then } \phi^{\prime}(t)=\mid f(t)^{-2} \cdot \int_{-\infty}^{\infty} \omega \cdot W_{f}(t, \omega) \cdot d \omega \\
-\Psi^{\prime}(\omega)=\mid F(\omega)^{-2} \int_{-\infty}^{\infty} t \cdot W_{f}(t, \omega) \cdot d t\end{array}$ \\
\hline (4) Moment properties & $\begin{array}{l}\int_{-\infty}^{\infty} \int_{-\infty}^{\infty} t^{n} W_{f}(t, \omega) d t d \omega=\int_{-\infty}^{\infty} t^{n}|f(t)|^{2} d t, \\
\int_{-\infty}^{\infty} \int_{-\infty}^{\infty} \omega^{n} W_{f}(t, \omega) d t d \omega=\int_{-\infty}^{\infty} \omega^{n}|F(\omega)|^{2} d \omega\end{array}$ \\
\hline
\end{tabular}

When $a=d=\cos \alpha, b=-c=\sin \alpha$, it becomes the FRFT. It also has the additivity property

$$
O_{F}^{\left(a_{2}, b_{2}, c_{2}, d_{2}\right)}\left(O_{F}^{\left(a_{1}, b_{1}, c_{1}, d_{1}\right)}(f(t))\right)=O_{F}^{(e, f, g, h)}(f(t))
$$

TABLE I

PROPERTIES OF WIGNER DISTRIBUTION FUNCTION (WDF)

where

$$
\left[\begin{array}{ll}
e & f \\
g & h
\end{array}\right]=\left[\begin{array}{ll}
a_{2} & b_{2} \\
c_{2} & d_{2}
\end{array}\right] \cdot\left[\begin{array}{ll}
a_{1} & b_{1} \\
c_{1} & d_{1}
\end{array}\right]
$$

We especially have

$$
O_{F}^{(d,-b,-c, a)}\left\lfloor O_{F}^{(a, b, c, d)}(f(t))\right\rfloor=f(t) .
$$

The applications of LCT are similar to those of the FRFT, but it is much more flexible, especially for optical system analysis and synthesis.

In this paper, we will use the following notation to represent the FRFT/LCT of $f(t)$ :

$$
\begin{aligned}
F_{\alpha}(u) & =O_{F}^{\alpha}(f(t)) \\
F_{(a, b, c, d)}(u) & =O_{F}^{(a, b, c, d)}(f(t)) .
\end{aligned}
$$

Many operations related to the Fourier transform have been generalized by the FRFT [3]. We list some of them in Table II. For the fractional correlation, we usually set $P_{3}$ as $-\pi / 2$ so that its formula can be simplified as

$$
O_{c o r r}^{P_{1}, P_{2}}(x(t), y(t))=\operatorname{IFT}\left(X_{P_{1}}(u) \cdot Y_{P_{2}}^{*}(u)\right) .
$$

Since the LCT is the generalization of FRFT, the fractional operations associated with the FRFT can be further generalized into the canonical operation by using the LCT. We list some canonical operations in Table III. Their applications are the same as their fractional counterparts.

At the end of Section II, we will list the relations between the WDF and the FRFT, the LCT, and some conventional operations in Table IV. They will be used in the following parts of this paper.

From the relations (1) and (2) in Table IV, we find that the FRFT just corresponds to the rotation operation for WDF, and the LCT just corresponds to the twisting operation for the WDF. From (5) and (6) in Table IV, we find the multiplication in the time domain corresponds to the convolution operation along the $w$-axis, and the conventional convolution operation corresponds to the convolution operation along $t$-axis for the WDF.

\section{RELATIONS BETWEEN THE WDF, AF, AND FRACTIONAL CONVOLUTION AND THEIR APPLICATIONS}

In Table IV, we have listed the relations between WDF and the operations of the FRFT, LCT, and conventional convolution, 


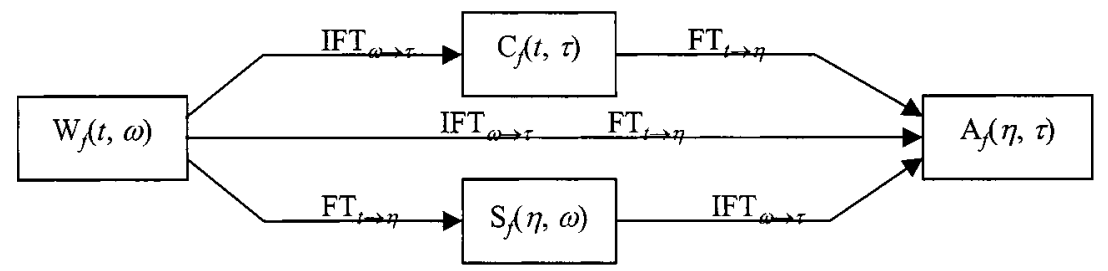

Fig. 1. Relations among the WDF, AF, signal and spectrum correlation function.

TABLE II

FRACTIONAL OPERATIONS RELATED TO THE FRFT

\begin{tabular}{|c|c|c|}
\hline $\begin{array}{l}\text { Fractional } \\
\text { operations }\end{array}$ & Formula & Applications \\
\hline $\begin{array}{l}\text { (1) Fractional } \\
\text { convolution } \\
{[15][17][18]}\end{array}$ & $O_{\text {conv }}^{\alpha}(x(t), y(t))=O_{F}^{-\alpha}\left(O_{F}^{\alpha}(x(t)) \cdot O_{F}^{\alpha}(y(t))\right)$ & $\begin{array}{l}\text { Filter design, beam } \\
\text { shaping, mask, } \\
\text { fractional Hilbert } \\
\text { transform } \\
\end{array}$ \\
\hline $\begin{array}{l}\text { (2) Fractional } \\
\text { correlation } \\
{[15][19][20][21]}\end{array}$ & $\begin{array}{l}\text { - type 1: } \\
O_{\text {corr }}^{P_{1}, P_{2} \cdot P_{3}}(x(t), y(t))=O_{F}^{P_{3}}\left(O_{F}^{P_{1}}(x(t)) O_{F}^{P_{2}}\left(y^{*}(-t)\right)\right) \\
\text { - type 2: (use in this paper) } \\
\quad O_{\text {corr }}^{P_{1}, P_{2}, P_{3}}(x(t), y(t))=O_{F}^{P_{3}}\left(X_{P_{1}}(u) \cdot Y_{P_{2}}^{*}(u)\right)\end{array}$ & $\begin{array}{c}\text { Space-variant } \\
\text { pattern recognition }\end{array}$ \\
\hline $\begin{array}{l}\text { (3) Fractional Hilbert } \\
\text { transform } \\
{[22][23][24]}\end{array}$ & $\begin{array}{l}O_{H l}^{\alpha, \phi}(x(t))=O_{F}^{-\alpha}\left(\left(e^{j \phi} s(u)+e^{-j \phi} s(-u)\right) O_{F}^{\alpha}(x(t))\right) \\
\text { where } s(u)=1 \text { when } u \geq 0, s(u)=0 \text { when } u<0\end{array}$ & Edge detection. \\
\hline
\end{tabular}

TABLE III

CANONICAL OPERATIONS RELATED to THE LCT

\begin{tabular}{|c|c|}
\hline (1) Canonical convolution & $O_{c o n v}^{(a, b, c, d)}(x(t), y(t))=O_{F}^{(d,-b,-c, a)}\left(O_{F}^{(a, b, c, d)}(x(t)) \cdot O_{F}^{(a, b, c, d)}(y(t))\right)$ \\
\hline (2) Canonical correlation & $O_{c o r r}(x(t), y(t))=O_{F}^{\left(a_{3}, b_{3}, c_{3}, d_{3}\right)}\left(X_{\left(a_{1}, b_{1}, c_{1}, d_{1}\right)}(u) \cdot Y_{\left(a_{2}, b_{2}, c_{2}, d_{2}\right)}^{*}(u)\right)$ \\
\hline $\begin{array}{l}\text { (3) Canonical Hilbert } \\
\text { transform }\end{array}$ & $O_{H l}^{(a, b, c, d), \phi}(x(t))=O_{F}^{(d,-b,-c, a)}\left(\left(e^{j \phi} s(u)+e^{-j \phi} s(-u)\right) O_{F}^{(a, b, c, d)}(x(t))\right)$ \\
\hline
\end{tabular}

correlation, and Hilbert transforms. In Sections III-V, we will discuss the relations between WDF and the fractional/canonical operations, and we will use these relations to discuss some applications and properties of FRFT/LCT.

\section{A. Relations Between Wigner Distribution Function and the Fractional/Canonical Convolution}

We first discuss the relation between the WDF and the fractional convolution. If $z(t)$ is the fractional convolution of $x(t)$ and $y(t)$

$$
\begin{aligned}
z(t)=x(t) & *_{\alpha} y(t) \\
& *_{\alpha}: \text { fractional convolution of order } \alpha
\end{aligned}
$$

then we can use the relation between the WDF and FRFT and the relation between the WDF and conventional convolution listed in Table IV and obtain the following [3], [14]-[16], [31], [32], [34].

\section{- Relation between Wigner distribution function and fractional convolution}

$$
\begin{aligned}
& W_{z}(t, \omega) \\
& =\int_{-\infty}^{\infty} W_{x}\left(t \cos ^{2} \alpha+\omega \sin \alpha \cos \alpha-\rho \sin \alpha\right. \\
& \left.t \sin \alpha \cos \alpha+\omega \sin ^{2} \alpha+\rho \cos \alpha\right) \\
& \quad \cdot W_{y}(t+\rho \sin \alpha, \omega-\rho \cos \alpha) \cdot d \rho .
\end{aligned}
$$

It can also be rewritten as

$$
\begin{aligned}
W_{z} & \left(\sigma \cdot \mathbf{t}_{1}+\mu \cdot \mathbf{w}_{1}\right) \\
= & \int_{-\infty}^{\infty} W_{x}\left(\sigma \cdot \mathbf{t}_{1}+\rho \cdot \mathbf{w}_{\mathbf{1}}\right) \\
& \cdot W_{y}\left(\sigma \cdot \mathbf{t}_{1}+\mu \cdot \mathbf{w}_{1}-\rho \cdot \mathbf{w}_{1}\right) \cdot d \rho
\end{aligned}
$$

where

$\sigma=t \cos \alpha+\omega \sin \alpha, \quad \mu=-t \sin \alpha+\omega \cos \alpha$

$\mathbf{t}_{1}=(\cos \alpha, \sin \alpha), \quad \mathbf{w}_{1}=(-\sin \alpha, \cos \alpha)$. 
TABLE IV

RELATIONS BETWEEN WDF AND FRFT, LCT, AND SOME CONVENTIONAL OPERATIONS

\begin{tabular}{|c|c|}
\hline Operations & Relations between WDF and the operations \\
\hline $\begin{array}{l}\text { (1) FRFT } \\
{[3][14][15][16]}\end{array}$ & $\begin{array}{l}\text { If } W_{f}(u, v), W_{F_{\alpha}}(u, v) \text { are the WDF of } f(t), F_{\alpha}(u) \text {, respectively, then } \\
W_{F_{\alpha}}(u, v)=W_{f}(\cos \alpha \cdot u-\sin \alpha \cdot v, \sin \alpha \cdot u+\cos \alpha \cdot v)\end{array}$ \\
\hline (2) LCT & 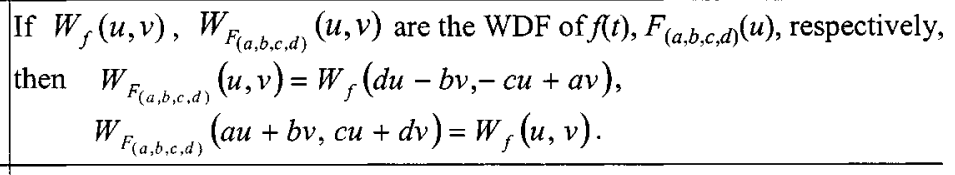 \\
\hline (3) $F_{\alpha}(u)$ & $\left|F_{\alpha}(u)\right|^{2}=\int_{-\infty}^{\infty} W_{f}(\cos \alpha \cdot u-\sin \alpha \cdot v, \sin \alpha \cdot u+\cos \alpha \cdot v) \cdot d v$ \\
\hline (4) $F_{(a, b, c, d)}(u)$ & $\left|F_{(a, b, c, d)}(u)\right|^{2}=\int_{-\infty}^{\infty} W_{f}(d \cdot u-b v,-c u+a v) \cdot d v$ \\
\hline $\begin{array}{l}\text { (5) Conventional } \\
\text { multiplication }\end{array}$ & If $z(t)=x(t) y(t)$, then $W_{z}(t, \omega)=\int_{\infty}^{\infty} W_{x}(t, \rho) W_{y}(t, \omega-\rho) \cdot d \rho$ \\
\hline $\begin{array}{l}\text { (6) Conventional } \\
\text { convolution }\end{array}$ & $\begin{array}{l}\text { If } z(t)=x(t) * y(t)=(2 \pi)^{-1} \int_{-\infty}^{\infty} x(\tau) y(t-\tau) d \tau, \text { then } \\
W_{z}(t, \omega)=\int_{-\infty}^{\infty} W_{x}(\rho, \omega) \cdot W_{y}(t-\rho, \omega) \cdot d \rho\end{array}$ \\
\hline $\begin{array}{l}\text { (7) Conventional } \\
\text { correlation }\end{array}$ & $\begin{array}{l}\text { If } z(t)=x(t) \otimes y(t)=(2 \pi)^{-1} \int_{-\infty}^{\infty} x(\tau+t) y^{*}(\tau) d \tau, \text { then } \\
W_{z}(t, \omega)=\int_{-\infty}^{\infty} W_{x}(\rho, \omega) \cdot W_{y}(-t+\rho, \omega) \cdot d \rho\end{array}$ \\
\hline $\begin{array}{l}\text { (8) Conventional } \\
\text { Hilbert } \\
\text { transform }\end{array}$ & 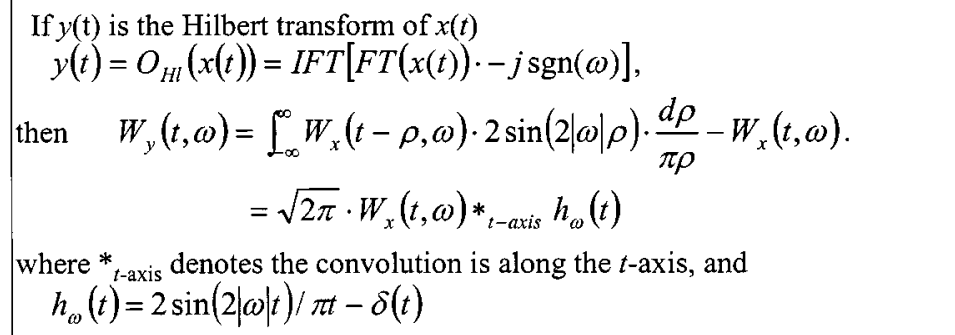 \\
\hline
\end{tabular}

This is the relation between WDF and the fractional convolution. Properties 5 and 6 in Table IV are just the special case that $\alpha=0$ and $\pi / 2$. From Table IV, we know that for the WDF, the multiplication operation just corresponds to the convolution along the direction parallel to the $\omega$-axis, and the conventional convolution corresponds to the convolution along the direction parallel to the $t$-axis. Now, from (15), we find that the fractional convolution corresponds to the convolution operation along the line that intersects the $\omega$-axis with the angle of $\alpha$ in the counterclockwise direction for WDF, as shown in Fig. 2(a).

For the case of canonical convolution, if $z(t)=$ $x(t) *(a, b, c, d) y(t)$, then we can also use the relations between the WDF and the FRFT and the conventional convolution listed in Table IV and obtain the following.

- Relation between Wigner distribution function and canonical convolution

$$
\begin{aligned}
W_{z}(t, \omega)= & \int_{-\infty}^{\infty} W_{x}(a d t+b d \omega-b \rho,-a c t-b c \omega+a \rho) \\
& \cdot W_{y}(t+b \rho, \omega-a \rho) d \rho .
\end{aligned}
$$
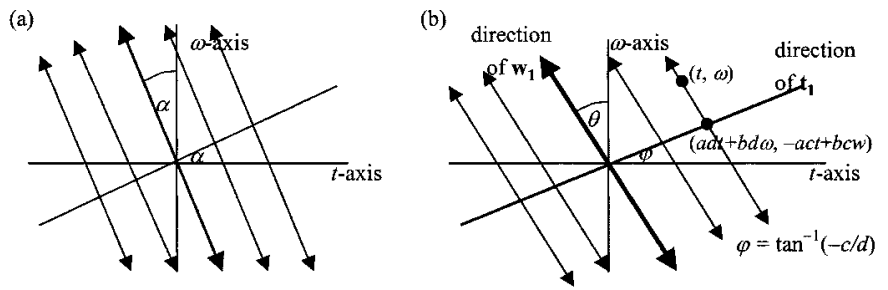

Fig. 2. Direction of convolution on WDF for (a) fractional convolution and (b) canonical convolution.

This equation can also be rewritten as

$$
\begin{aligned}
& W_{z}\left(\sigma \cdot \mathbf{t}_{1}+\mu \cdot \mathbf{w}_{1}\right) \\
& =\int_{-\infty}^{\infty} W_{x}\left(\sigma \cdot \mathbf{t}_{1}+\rho \cdot \mathbf{w}_{1}\right) \\
& \quad \cdot W_{y}\left(\sigma \cdot \mathbf{t}_{1}+\mu \cdot \mathbf{w}_{1}-\rho \cdot \mathbf{w}_{1}\right) \cdot d \rho
\end{aligned}
$$

where

$$
\begin{aligned}
\sigma & =a t+b \omega, & \mu & =c t+d \omega \\
\mathbf{t}_{\mathbf{1}} & =(d,-c), & \mathbf{w}_{\mathbf{1}} & =(-b, a) .
\end{aligned}
$$

We find, for the WDF, that the canonical convolution corresponds to the convolution along the line that intersects 
the $\omega$-axis with the angle of $\tan ^{-1}(b / a)$ in the counterclockwise direction, as shown in Fig. 2(b).

From (18), we find that the parameters of LCT will affect the canonical convolution from the following ways.

1) The value of $\boldsymbol{b} / \boldsymbol{a}$ will affect the direction of convolution.

This is because $\mathbf{w}_{\mathbf{1}}=(-b, a)$ is the direction of the convolution.

2) The value of $\boldsymbol{c} / \boldsymbol{d}$ will shear the Wigner distribution function of the result.

This is because the direction of $\mathbf{t}_{1}=(d,-c)$ in Fig. 2(b) is determined by $c / d$. For example, suppose we use the same values of $a, b$ as in Fig. 2(b), but the value of $c, d$ is changed to $p, q$. Then, the direction of $\mathbf{t}_{1}$ is changed [we denote the new direction $(p, q)$ to $\mathbf{t}_{\mathbf{2}}$, but the direction of $\mathbf{w}_{1}$, i.e., the direction of convolution, will remain unchanged]. To see why the WDF is sheared, we suppose that

$$
\begin{aligned}
z(t) & =x(t) *_{(a, b, c, d)} y(t) \\
z_{1}(t) & =x(t) *_{(a, b, p, q)} y(t) .
\end{aligned}
$$

Then, the WDF of $z(t)$ is the same as (18) and (19), and the WDF of $z_{1}(t)$ is

$$
\begin{aligned}
& W_{z 1}\left(\sigma \cdot \mathbf{t}_{\mathbf{2}}+\mu_{2} \cdot \mathbf{w}_{1}\right) \\
& =\int_{-\infty}^{\infty} W_{x}\left(\sigma \cdot \mathbf{t}_{\mathbf{2}}+\rho \cdot \mathbf{w}_{\mathbf{1}}\right) \\
& \quad \cdot W_{y}\left(\sigma \cdot \mathbf{t}_{\mathbf{2}}+\mu_{2} \cdot \mathbf{w}_{1}-\rho \cdot \mathbf{w}_{1}\right) \cdot d \rho
\end{aligned}
$$

where $\sigma=a t+b \omega, \mu_{2}=p t+q \omega, \mathbf{t}_{2}=(q,-p)$, $\mathbf{w}_{1}=(-b, a)$. Then, from the relation that

$$
\sigma \cdot \mathbf{t}_{1}+\mu \cdot \mathbf{w}_{1}=\sigma \cdot \mathbf{t}_{2}+\mu_{2} \cdot \mathbf{w}_{1}=(t, \omega)
$$

after some computation, we can prove

$$
W_{z 1}\left(\sigma \cdot \mathbf{t}_{\mathbf{2}}+\left(2 \mu_{2}-\mu\right) \cdot \mathbf{w}_{1}\right)=W_{z}\left(\sigma \cdot \mathbf{t}_{\mathbf{1}}+\mu \cdot \mathbf{w}_{\mathbf{1}}\right) .
$$

Thus, if $b / a$ is unchanged, and only the value of $c / d$ is changed, then the WDF of the canonical convolution result is just sheared along the direction of $\mathbf{w}_{\mathbf{1}}=(-b, a)$ (see Fig. 3).

Most of the applications of the LCT, such as the filter design, are associated with the canonical convolution, and from the above discussion, only the values of $b / a$ and $c / d$ will affect the results of canonical convolution; therefore, only the values of $b / a$ and $c / d$ are important for the LCT. Besides, since the value of $b / a$ affects the direction of convolution for the WDF, and the value of $c / d$ just shears the WDF, we can conclude that the value of $b / a$ is more important than the value of $c / d$. The LCT has four parameters, and for the applications about LCT, it seems we have to adjust four the parameters, but in fact, we have the following.

1) We can just control the values of $\boldsymbol{b} / \boldsymbol{a}$ (it is always enough to satisfy our requirement.)

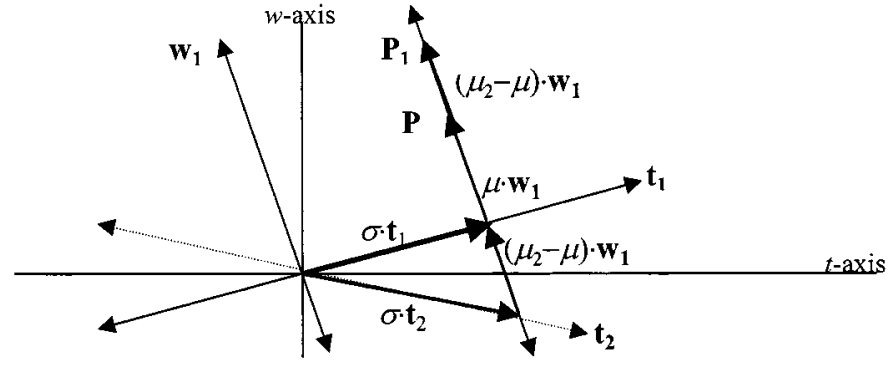

Fig. 3. Value of $W_{z 1}(t, \omega)$ [defined as (21)] at the location $\mathbf{P}_{\mathbf{1}}$ will be the same as the value $W_{z}(t, \omega)$ at the location $\mathbf{P}$.

2) Sometimes, we may also control the values of $\boldsymbol{c} / \boldsymbol{d}$.

3) It is always unnecessary to control other parameters.

\section{B. Applications of the Relations Between WDF and Fractional/Canonical Convolution for Filter Design}

From the relations between WDF and the fractional/canonical convolution, we can discuss the performance and the design methods for the applications associated with the fractional/canonical convolution.

Many papers [15], [24]-[27] discuss the use of the filter designed by the FRFT or LCT to remove noise or distortion. The effect of the filter designed by the LCT can be written in the following equation:

$$
y(t)=O_{F}^{(d,-b,-c, a)}\left(H(u) \cdot O_{F}^{(a, b, c, d)}(x(t))\right) .
$$

It is just a special case of canonical convolution.

There are many possible types of canonical filter. The simplest is the pass-stopband filter. The transform function of the pass-stopband filter is

$$
H(u)=\Pi\left(\left(u-u_{0}\right) / B\right) .
$$

That is

$$
\begin{aligned}
& H(u)=1, \quad \text { when } u_{0}-B / 2<u<u_{0}+B / 2 \\
& H(u)=0, \quad \text { otherwise. }
\end{aligned}
$$

The WDF of $H(u)$ is

$$
\begin{aligned}
W_{H}(u, v)= & (\pi v)^{-1} \sin \left(v \cdot\left(2 B-\left|4\left(u-u_{0}\right)\right|\right)\right) \\
& \cdot \Pi\left(\left(u-u_{0}\right) / B\right)
\end{aligned}
$$

and if $h(t)=O_{F}^{(d,-b,-c, a)}(H(u))$, then the WDF of $h(t)$ is

$$
\begin{aligned}
W_{h}(t, \omega)= & \frac{\sin \left((c t+d w)\left(2 B-\left|4\left(a t+b \omega-u_{0}\right)\right|\right)\right)}{\pi(c t+d \omega)} \\
& \cdot \Pi\left(\frac{a t+b \omega-u_{0}}{B}\right) .
\end{aligned}
$$


In addition, from (17), the WDF of the filter output $y(t)$ is

$$
\begin{aligned}
W_{y}(t, \omega) & \int_{-\infty}^{\infty} W_{x}(a d \cdot t+b d \cdot \omega-b \cdot \rho,-a c \cdot t-b c \cdot \omega+a \cdot \rho) \\
& \cdot \frac{\sin \left((c t+d \omega-\rho)\left(2 B-\mid 4\left(a t+b \omega-u_{0} \mid\right)\right)\right.}{\pi(c t+d \omega-\rho)} \\
& \cdot \Pi\left(\frac{\left(a t+b \omega-u_{0}\right)}{B}\right) \cdot d \rho \\
= & \int_{-\infty}^{\infty} W_{x}(d \eta-b \rho,-c \eta+a \rho) \\
& \cdot \frac{\sin \left((c t+d \omega-\rho)\left(2 B-\mid 4\left(\eta-u_{0} \mid\right)\right)\right.}{\pi(c t+d \omega-\rho)} \\
& \cdot \Pi\left(\frac{\left(\eta-u_{0}\right)}{B}\right) d \rho
\end{aligned}
$$

where $\eta=a t+b w$. Since

$\Pi\left(\left(\eta-u_{0}\right) / B\right)=0 \quad$ when $\eta<u_{0}-B / 2$ or $\eta>u_{0}+B / 2$

so $W_{x}(t, \omega)$ will have no effect on the WDF of $y(t)$ when

$$
u_{0}-B / 2<a t+b \omega<u_{0}+B / 2 \text {. }
$$

That is, if the WDF of the undesired part of $x(t)$ is outside the region of (30), then we can filter out the undesired part by the method as (24) and (25). Thus, from the WDF of the received signal (such as Fig. 4), we can design the pass-stopband canonical filter. In fact, we have the following.

1) The parameter $a / b$ can control the slope of the cutoff line on WDF.

2) The parameters $u_{0}, B$ can control the location of the pass region on WDF.

For example, suppose the WDF of the received signal can be drawn as Fig. 4. The dotted regions indicate the locations where

$$
W_{x}(t, \omega)>T, \quad \text { where } T \text { is the threshold. }
$$

We also suppose that the desired and undesired parts can be separated by two parallel cutoff lines. Then, we can choose the parameters $a, b, c, d, u_{0}$, and $B$ in (24) and (25) of the canonical filter as

$$
\begin{gathered}
a / b=\omega_{1} / t_{1} \quad \text { (for the case of fractional filter } \\
\left.\alpha=\cot ^{-1}\left(\omega_{1} / t_{1}\right)\right) \\
u_{0}=a\left(t_{0}+t_{1}\right) / 2, \quad B=\left|a\left(t_{1}-t_{0}\right)\right| .
\end{gathered}
$$

We give an example in Fig. 5 to illustrate the above idea. Here, we use $x(t)$ as the original input signal, as shown in Fig. 5(a):

$$
x(t)=2 \cdot \exp \left(-0.2 \cdot t^{2}\right) \cdot \Pi(t / 6) .
$$

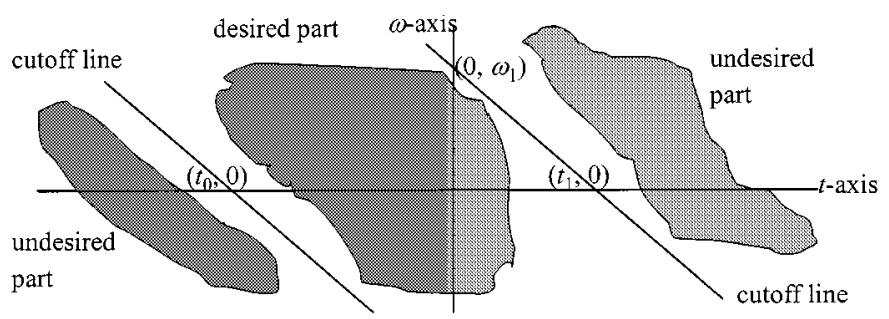

Fig. 4. Using WDF to filter out the undesired signal by pass-stopband canonical filter.

We suppose that $x(t)$ is interfered (here, "interfered" just means linear addition) by the chirp and modulated Gaussian function

$$
\begin{aligned}
n(t)= & 0.5 \cdot \exp \left(j \cdot 0.3 \cdot(t+8.6)^{2}\right)+\exp (-j \cdot 3 t) \\
& \cdot \exp \left(-0.1 \cdot(t-7)^{2}\right) .
\end{aligned}
$$

The interfered signal is shown in Fig. 5(b). It is hard to use the original convolution to remove the undesired function, but we can use the canonical convolution. We can first calculate the WDF of $x(t)+n(t)$ and then use the WDF to determine the parameters of the canonical pass-stopband filter. We draw the WDF of $x(t)+n(t)$ in Fig. 6(a). The central ellipse in Fig. 6(a) is the WDF of $x(t)$, and the left line and the right ellipse are the WDF of $n(t)$. Others are cross terms.

Then we can use two parallel lines to separate the desired and the undesired part on the WDF of $x(t)+n(t)$ (we do not consider the cross terms), as shown in Fig. 6(b). The left cut-off line passes through $(-6,0)$ and $(0,3.6)$, and the right cut-off line passes through $(6,0)$ and $(10,2.4)$. They both have the slope of 0.6. Then, from (32), we obtain $a / b=-0.6, u_{0}=0$, and $B=7.2$. We can fix the values of the parameters $b, c$, and $d$ of the LCT as $\{1,-1,0\}$; then, $a=-0.6$. Therefore, the overall system of the pass-stopband canonical filter can be designed as

$$
\begin{aligned}
x_{o}(t)= & O_{F}^{(0,-1,1,-0.6)} \\
& \cdot\left(\Pi(u / 7.2) \cdot O_{F}^{(-0.6,1,-1,0)}(x(t)+n(t))\right) .
\end{aligned}
$$

We plot the LCT with parameters $\{-0.6,1,-1,0\}$ for $x(t)+$ $n(t)$ in Fig. 5(c) and plot the filter output in Fig. 5(d). We find that the undesired signal is removed by the canonical filter.

There are also other types of canonical filter, such as the optimal canonical filter [26]. From the discussion in Section III-A, we know only the value of $a / b$ is important for canonical convolution and, hence, for the canonical filter. Thus, for any type of canonical filter, we just have to adjust the value of $a / b$ and the transfer function.

\section{Information Contained in the Phase for the FRFT/LCT}

It is well-known that in the FT, the phase is more important than the amplitude for the image signal. Suppose that $G(k, h)$ is the 2-D FT of an image signal $g(x, y)$

$$
G(k, h)=\mathrm{FT}_{2 \mathrm{D}}(g(x, y))
$$




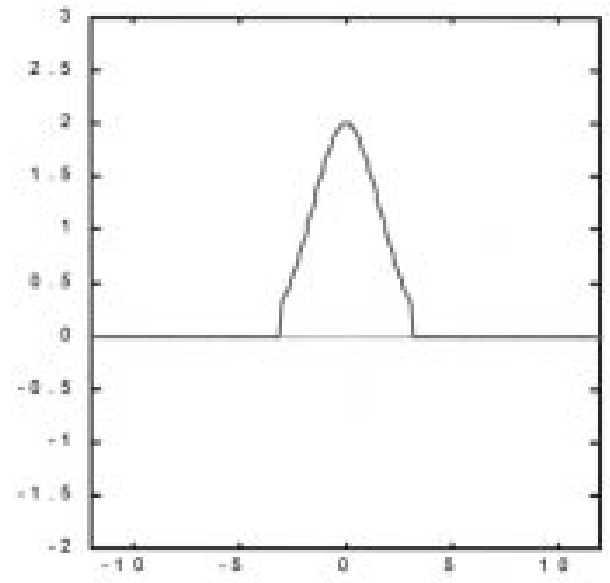

(a)

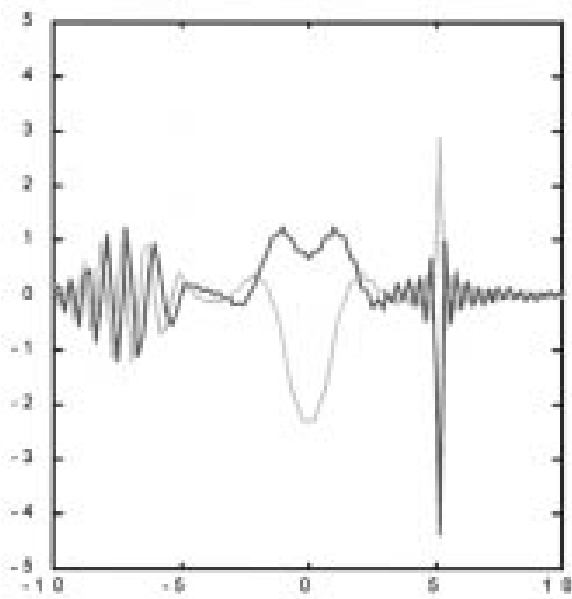

(c)

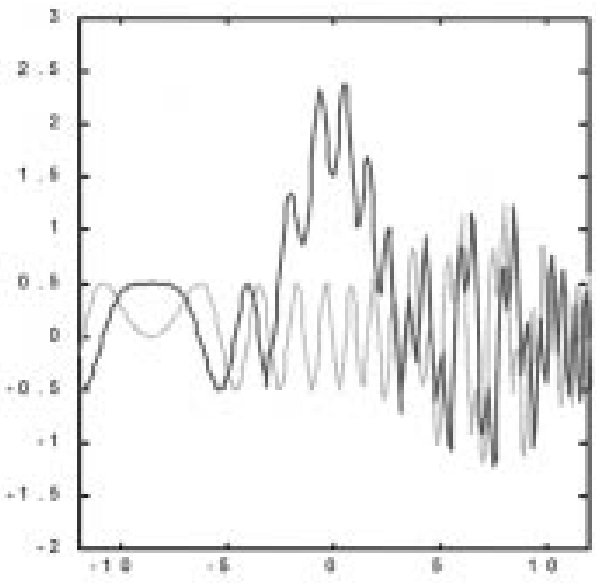

(b)

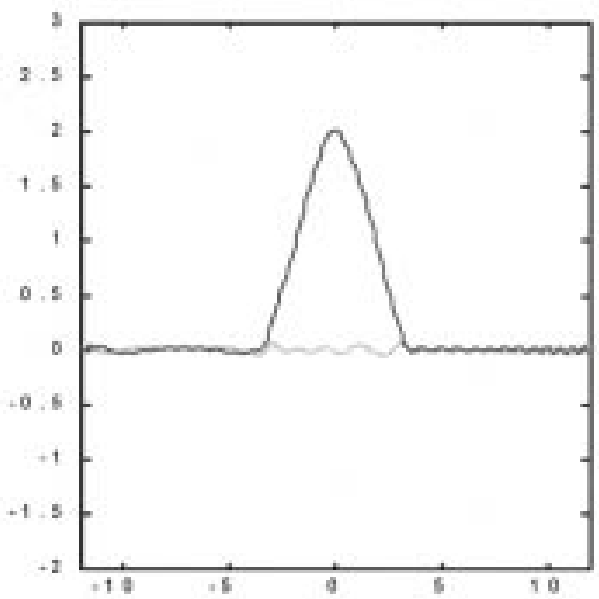

(d)

Fig. 5. Using canonical convolution to filter out the undesired signal. (a) Original input. (b) Interfered by the undesired signal. (c) LCT of interfered input. (d) Filter output.

If we separate $G(k, h)$ into the amplitude part and the phase part

$$
\begin{array}{r}
G(k, h)=|G(k, h)| \cdot P(k, h) \\
\text { where } P(k, h)=G(k, h) /|G(k, h)|
\end{array}
$$

then we can obtain the edges of $g(x, y)$ by doing the inverse 2-D FT for the phase $P(k, h)$, but if we do the inverse 2-D FT for the amplitude $|G(k, h)|$, then we cannot see any information.

Then, we will ask a question: For the FRFT and LCT, is the phase still more important than the amplitude? We will do some experiments to find out. We do the FRFT with parameter $\alpha=$ $3 \pi / 8, \pi / 4, \pi / 8$, and $\pi / 90$, separate the amplitude and phase parts, and then do the inverse FRFT for the amplitude parts and phase parts. We show the results in Figs. 7 and 8.

There are some phenomena that can be observed from the experiments. We find, when $\alpha$ (or $|b / a|$ ) is large (such as the case that $\alpha=3 \pi / 8$ ), the phase-only image will also show the edges of the original image. It is similar to the case of original FT. In addition, when $\alpha$ (or $|b / a|$ ) becomes smaller (such as the case that $\alpha=\pi / 4, \pi / 8$ ), the low-frequency components also appear for the phase-only image, and the edges are not as obvious as the case in which $\alpha$ (or $|b / a|$ ) is large.

For the amplitude-only image, except for the case that $\alpha$ (or $|b / a|)$ is very small, there will be no information seen. Therefore, for the FRFT/LCT, the phase is usually more important than the amplitude, except for the case in which $|\alpha|$ (or $|b / a|)$ is very small.

We can illustrate the above by the WDF. For convenience, we will simply discuss the phase-only image and consider the one-dimensional (1-D) case. Then, for the original FT, (36) and (37) are simplified to

$$
\begin{aligned}
& G(\omega)=\mathrm{FT}(g(t))=|G(\omega)| \cdot P(\omega) \\
& P(\omega)=G(\omega) /|G(\omega)| .
\end{aligned}
$$




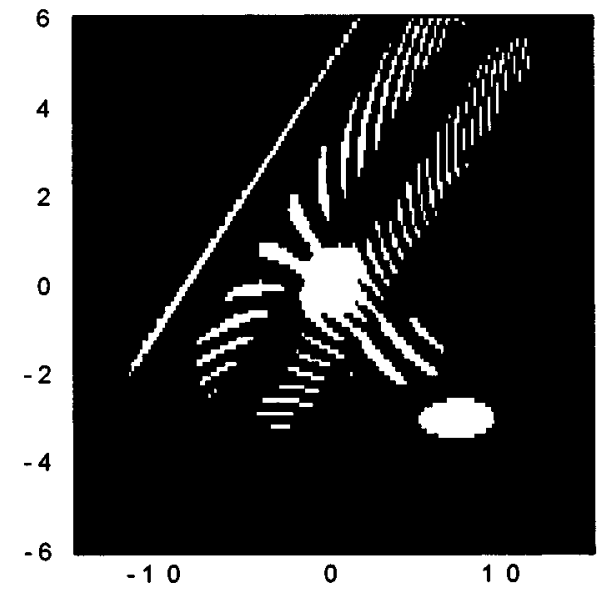

(a)

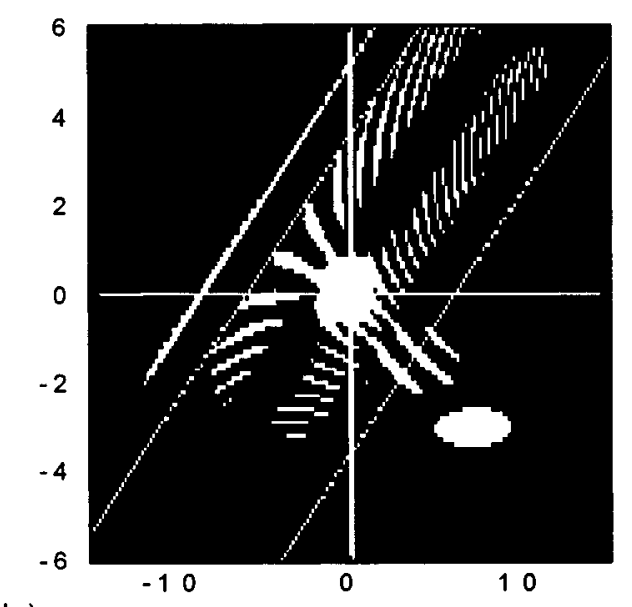

(b)

Fig. 6. (a) WDF of $x(t)+n(t)$. (b) WDF of $x(t)+n(t)$, and the locations of $t$-axis, $\omega$-axis, and the two cutoff lines.

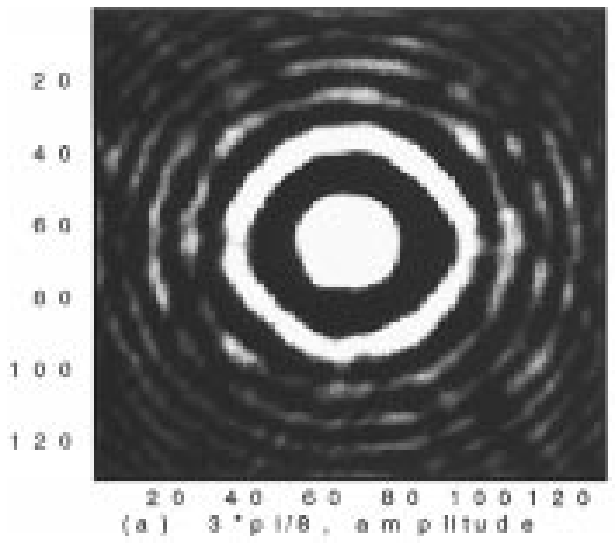

(a)

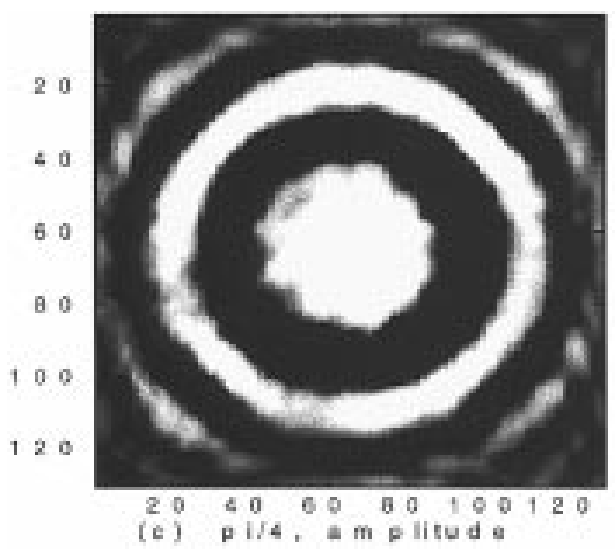

(c)

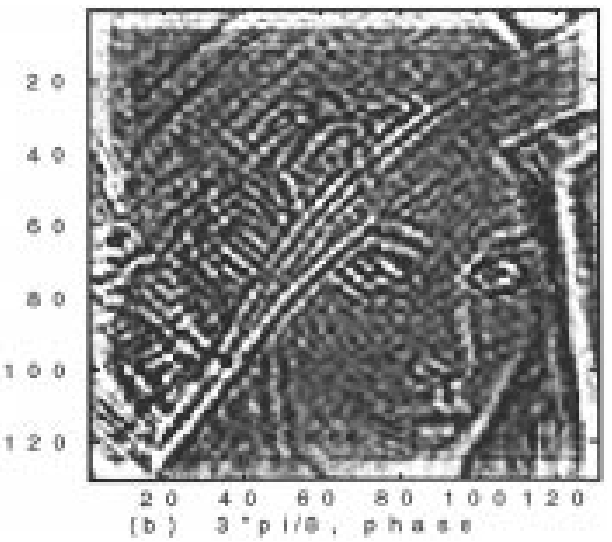

(b)

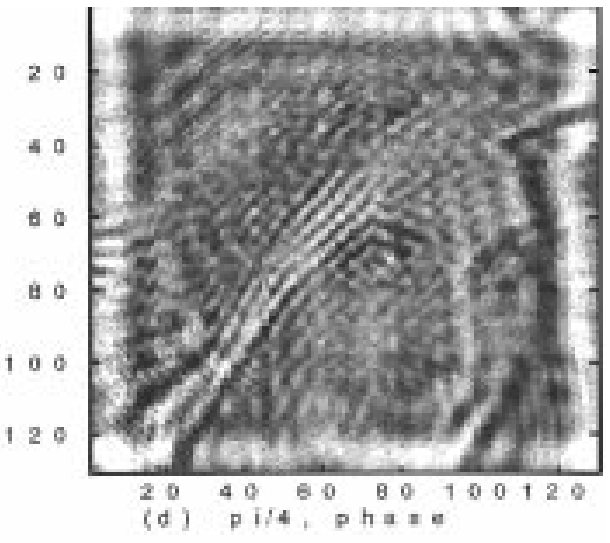

(d)

Fig. 7. Inverse 2-D FRFT of the amplitude/phase parts of Lena image's 2-D FRFT when $\alpha=3 \pi / 8$ and $\alpha=\pi / 4$.

For the cases of FRFT/LCT, they will be changed to

$$
\begin{aligned}
G_{(a, b, c, d)}(u) & =O_{F}^{(a, b, c, d)}(g(t)) \\
& =\left|G_{(a, b, c, d)}(u)\right| \cdot P_{(a, b, c, d)}(u) \\
P_{(a, b, c, d)}(u) & =G_{(a, b, c, d)}(u) /\left|G_{(a, b, c, d)}(u)\right| .
\end{aligned}
$$

From the projection property of the WDF, we can obtain $|G(\omega)|$ from the integration of $W_{g}(t, \omega)$ along the $t$-axis:

$$
|G(\omega)|=\left(\int_{-\infty}^{\infty} W_{g}(t, \omega) \cdot d t\right)^{1 / 2}
$$

Since for most of the signals, including image signals, the lowfrequency components are usually greater than the high compo- 


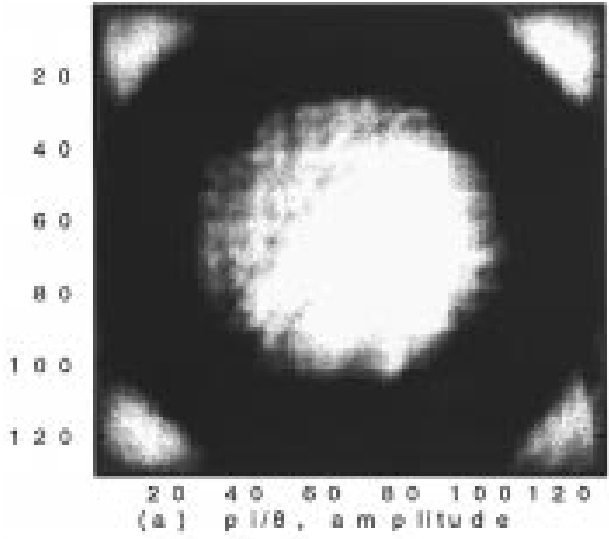

(a)

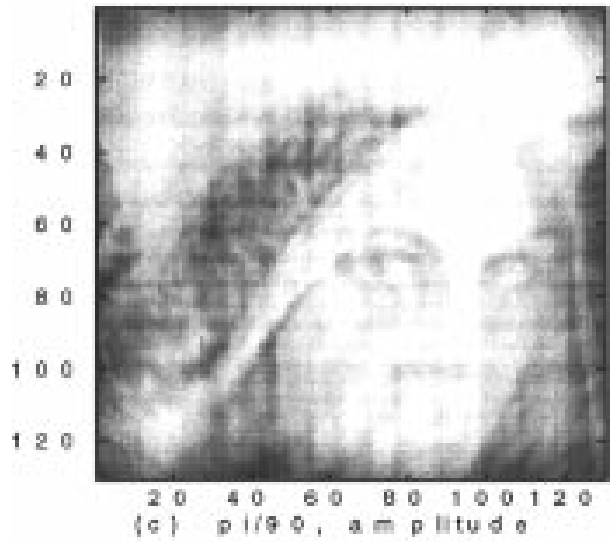

(c)

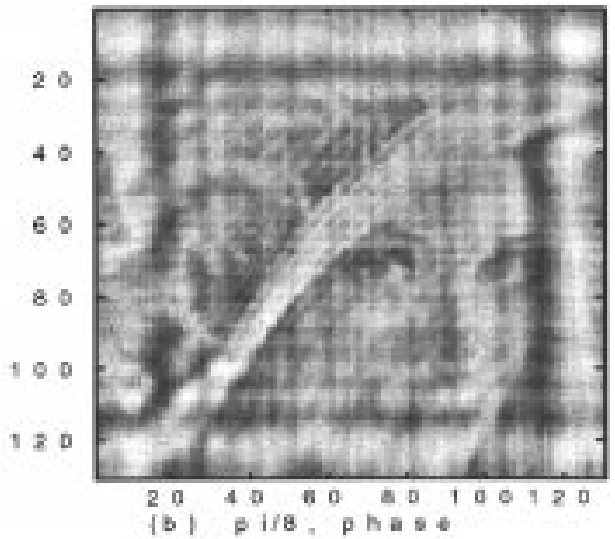

(b)

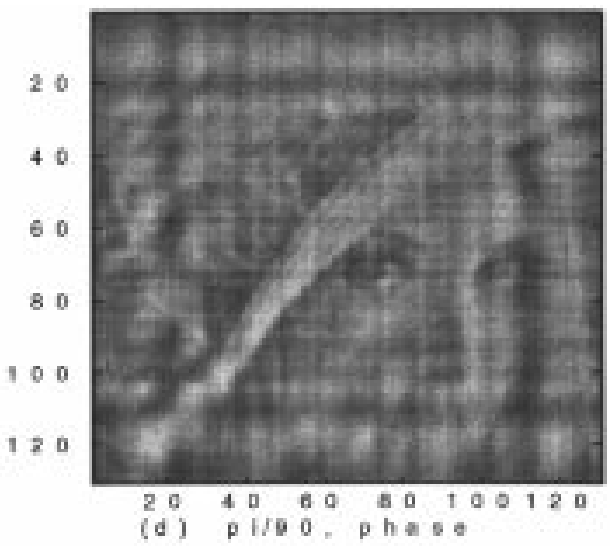

(d)

Fig. 8. Inverse 2-D FRFT of the amplitude/phase parts of Lena image's 2-D FRFT when $\alpha=\pi / 8$ and $\alpha=\pi / 90$.

nents; therefore, the inequalities are often satisfied as follows.

$$
\begin{aligned}
& W_{g}\left(\omega_{1}, t\right)>W_{g}\left(\omega_{2}, t\right), \quad \text { when } \omega_{2}>\omega_{1} \\
& \int_{-\infty}^{\infty} W_{g}\left(\omega_{1}, t\right) d t>\int_{-\infty}^{\infty} W_{g}\left(\omega_{2}, t\right) d t \\
& \left|G\left(\omega_{1}\right)\right|>\left|G\left(\omega_{2}\right)\right| \\
& 1 /\left|G\left(\omega_{1}\right)\right|<1 /\left|G\left(\omega_{2}\right)\right|, \quad \text { when } \omega_{2}>\omega_{1} .
\end{aligned}
$$

In (38), $1 /|G(\omega)|$ will act as a transfer function of highpass filter. Thus, $P(\omega)$ will amplify the high-frequency components of $G(\omega)$ and attenuate the low-frequency components. Edges usually belong to the high-frequency components. Therefore, after we do the inverse FT for $P(\omega)$, the edges of the original signals will be emphasized, and other components are attenuated. This is why for the original FT, the phase-only image shows the edges of the original image.

In addition, for the case of LCT, from the projection property of LCT (which is listed in Table IV), we obtain

$$
\left|G_{(a, b, c, d)}(u)\right|=\left(\int_{-\infty}^{\infty} W_{g}(d u-b v,-c u+a v) \cdot d v\right)^{1 / 2} .
$$

That is, $\left|G_{(a, b, c, d)}(u)\right|$ can be obtained by integrating $W_{g}(t, \omega)$ along the direction of $(-b, a)$. Since, when $a \neq 0$, the integration direction no longer parallels the $t$-axis, $\left|G_{(a, b, c, d)}(u)\right|$ will also contain some low-frequency components, even when $u$ is

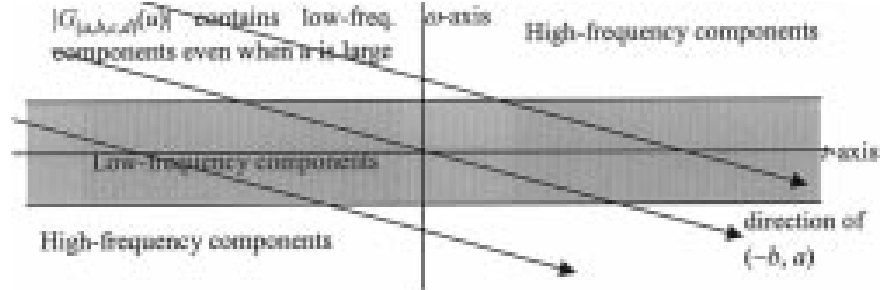

Fig. 9. Projection direction to obtain $\left|G_{a, b, c, d)}(u)\right|$ from $W_{g}(t, \omega)$.

large, and $\left|G_{(a, b, c, d)}(u)\right|$ will also contain some high-frequency components when $u$ is small, as in Fig. 9.

Thus, although the inequality of (44) is usually still satisfied, the ratio between the amplitude for higher value of $u_{2}$ and lower value of $u_{1}$ will be less

$$
\begin{aligned}
& \left|G_{(a, b, c, d)}\left(u_{1}\right)\right| /\left|G_{(a, b, c, d)}\left(u_{2}\right)\right| \\
& \quad<\left|G\left(u_{1}\right)\right| /\left|G\left(u_{2}\right)\right|, \quad \text { when } u_{2}>u_{1} .
\end{aligned}
$$

In the case that $a \neq 0$, although in the edges are usually still emphasized for the phase-only image, the edges will be smoother than the case where $a=0$, and the low-frequency components are not attenuated as seriously as the case where $a=0$. Therefore, when the value of $|b / a|$ is in the middle, we can still see both the high- and the low-frequency components of the original image. 
In addition, when $|b / a|$ is very small, then $(-b, a)$ is near the direction parallel to the $\omega$-axis, and

$$
\begin{aligned}
\left|G_{(a, b, c, d)}(u)\right| & =\left(\int_{-\infty}^{\infty} W_{g}(d u-b v,-c u+a v) \cdot d v\right)^{1 / 2} \\
& \approx\left(\int_{-\infty}^{\infty} W_{g}(d u,-c u+v) \cdot d v\right)^{1 / 2} \\
\left|G_{(a, b, c, d)}(u)\right| & \approx\left(\int_{-\infty}^{\infty} W_{g}(d u, v) \cdot d v\right)^{1 / 2} \\
& =g(d t) \approx g\left(a^{-1} t\right), \quad \text { when }|b / a| \text { is small }
\end{aligned}
$$

and

$$
\begin{aligned}
g_{k}(t) & =O_{F}^{(d,-b,-c, a)}\left(\left|G_{(a, b, c, d)}^{-1}(u)\right|\right) \\
& \approx O_{F}^{\left(a d, b a^{-1},-c a, 1\right)}\left(O_{F}^{\left(a^{-1}, 0,0, a\right)} g^{-1}\left(a^{-1} t\right)\right) \\
& \approx g^{-1}(t) \\
g_{p}(t) & =g(t) *_{(a, b, c, d)} g_{k}(t) \\
& \approx g(t) \cdot g_{k}(t) \approx g(t) \cdot g^{-1}(t) .
\end{aligned}
$$

When $|b / a|$ is very small, the phase-only image seems to have smoothed the original image, as we see from Fig. 8(d). When $|b / a| \rightarrow 0$, then $g_{p}(t) \approx 1$ for all $t$.

Thus, we can conclude the following.

a) When $|b / a|$ is large, then the phase-only image seems like the original image passing through the highpass (edges detection) filter.

b) When $|b / a|$ is in the middle, then the phase-only image is similar to the original image passing through the fractional/canonical filter between highpass and allpass.

c) When $|b / a|$ is small, then the phase-only image is like the original image passing through the smoother filter.

\section{Relations between the WDF and the Fractional/Canonical Hilbert Transform, Fractional Beam Shaping}

Before discussing the case of fractional and canonical Hilbert transform, we need to discuss the WDF of $\exp (j \phi) u(f)+\exp (-j \phi) u(-f)$ first. If

$$
H_{\phi}(f)=e^{j \phi} u(f)+e^{-j \phi} u(-f)
$$

then

$$
\begin{aligned}
& H_{\phi}\left(f+\frac{\tau}{2}\right) \cdot H_{\phi}^{*}\left(f-\frac{\tau}{2}\right) \\
& \quad=e^{-j 2 \phi} u(-\tau-2|f|)+\operatorname{rect}\left(\frac{\tau}{4|f|}\right)+e^{j 2 \phi} u(\tau-2|f|) .
\end{aligned}
$$

From a trick of integration [30]

$$
\begin{aligned}
\int_{-\infty}^{\infty} u(\tau-a) \cdot e^{-j \omega \tau} d \tau \\
=\lim _{N \rightarrow \infty} \int_{a}^{\infty} e^{-\tau / N} e^{-j \omega \tau} d \tau \\
=e^{-j \omega \cdot a} / j \omega \quad \text { when } \omega \neq 0
\end{aligned}
$$

we obtain the WDF of $H_{\phi}(f)$ as

$$
\begin{aligned}
W_{H_{\phi}}(t, \omega)= & (\pi \omega)^{-1} \cdot[\sin (2|t| \omega)+\sin (2 \phi-2|t| \omega)] \\
& +\cos 2 \phi \cdot \delta(\omega) .
\end{aligned}
$$

Thus, the WDF of $h l_{\phi}(t)=O_{F}^{-\alpha}\left(h_{\phi}(\omega)\right)$ is

$$
\begin{aligned}
& W_{h l_{\phi}}(u, v) \\
& =\cos 2 \phi \cdot \delta(-u \sin \alpha+v \cos \alpha) \\
& \quad+\frac{\sin (2|u \cos \alpha+v \sin \alpha|(-u \sin \alpha+v \cos \alpha))}{\pi(-u \sin \alpha+v \cos \alpha)} \\
& \quad+\frac{\sin (2 \phi-2|u \cos \alpha+v \sin \alpha|(-u \sin \alpha+v \cos \alpha))}{\pi(-u \sin \alpha+v \cos \alpha)} .
\end{aligned}
$$

Then from (14) and (55), we find that if

$$
y(t)=O_{H l}^{\alpha, \phi}(x(t))=x(t) *_{\alpha} h l_{\phi}(t)
$$

then

$$
\begin{aligned}
& W_{y}(t, \omega) \\
& =\frac{1}{\pi} \int_{-\infty}^{\infty} W_{x}\left(t \cos ^{2} \alpha+\omega \sin \alpha \cos \alpha-\rho \sin \alpha\right. \\
& \left.\quad \cdot t \sin \alpha \cos \alpha+\omega \sin ^{2} \alpha+\rho \cos \alpha\right) \\
& \quad \cdot(\omega \cos \alpha-t \sin \alpha-\rho)^{-1} \\
& \quad \cdot[\sin (2(\omega \cos \alpha-t \sin \alpha-\rho)|t \cos \alpha+\omega \sin \alpha|) \\
& \quad-\sin (2 \phi+2(\omega \cos \alpha-t \sin \alpha-\rho)|t \cos \alpha+\omega \sin \alpha|)] \\
& \quad \cdot d \rho+\cos 2 \phi \cdot W_{x}(t, \omega) .
\end{aligned}
$$

For the case of canonical Hilbert transform, if $y(t)=$ $O_{H l}^{(a, b, c, d), \phi}(x(t))$, then

$$
\begin{aligned}
& W_{y}(t, \omega) \\
& =\frac{1}{\pi} \int_{-\infty}^{\infty} W_{x}(a d \cdot t+b d \cdot \omega-b \cdot \rho \\
& \quad \cdot-a c \cdot t-b c \cdot \omega+a \cdot \rho) \cdot(c t+d \omega-\rho)^{-1} \\
& \quad \cdot[\sin (2(c t+d \omega-\rho) \cdot|a t+b \omega|) \\
& \quad \quad-\sin (2 \phi+2(c t+d \omega-\rho) \cdot|a t+b \omega|)] \\
& \quad \cdot d \rho+\cos 2 \phi \cdot W_{x}(t, \omega) .
\end{aligned}
$$

The performance of canonical Hilbert transform can be illustrated by its relation with the WDF. We note that when $\phi=\pi / 2$, (58) can be rewritten as

$$
\begin{aligned}
& W_{y}\left(\sigma \cdot \mathbf{t}_{1}+\mu \cdot \mathbf{w}_{1}\right) \\
& \quad=\int_{-\infty}^{\infty} W_{x}\left(\sigma \cdot \mathbf{t}_{1}+\rho \cdot \mathbf{w}_{1}\right) h_{(a, b, c, d), \phi}^{\sigma}(\mu-\rho) d \rho \\
& \quad-W_{x}\left(\sigma \cdot \mathbf{t}_{1}+\mu \cdot \mathbf{w}_{1}\right)
\end{aligned}
$$

where $\sigma, \mu, \mathbf{t}_{1}$, and $\mathbf{w}_{1}$ are defined as (19), and $h_{(a, b, c, d), \phi}^{\sigma}(\mu)$ is a 2-D to 1-D function as in

$$
h_{(a, b, c, d), \phi}^{\sigma}(\mu)=2 \mu^{-1} \sin (2 \mu \cdot|\sigma|) .
$$

That is, $W_{x}(t, w)$ convolves with $h_{(a, b, c, d), \phi}^{\sigma}(\mu)$ along the direction of $\mathbf{w}_{1}=(-b, a)$. We note that when $|\sigma|$ is low, (60) acts 
as a lowpass filter, and when $|\sigma|$ is high, (60) acts as an allpass filter. Therefore, after the canonical Hilbert transform, when the value of $|\sigma|$ is small, $W_{x}\left(\sigma \cdot \mathbf{t}_{1}, \mu \cdot \mathbf{w}_{1}\right)$ are destroyed by the LP filter, and when the value of $|\sigma|$ is large, $W_{y}\left(\sigma \cdot \mathbf{t}_{1}, \mu \cdot \mathbf{w}_{1}\right)$ will be almost the same as $W_{x}\left(\sigma \cdot \mathbf{t}_{1}, \mu \cdot \mathbf{w}_{1}\right)$.

For the conventional Hilbert transform, $\mathbf{t}_{1}=(0,1)$ and $\mathbf{w}_{1}=(-1,0)$; therefore, the smaller or larger value of $|\sigma|$ means the smaller or large value of $|\omega|$, respectively. Therefore, after the conventional Hilbert transform, the low-frequency components are destroyed, and the high-frequency components have almost remained the same. Thus, the conventional Hilbert transform acts as a highpass filter and can be used for edge detection.

However, for the canonical Hilbert transform, since $\mathbf{t}_{1}=$ $(d,-c)$ and $\mathbf{w}_{\mathbf{1}}=(-b, a)$, the regions with a smaller value of $|\sigma|$ and the regions with a higher value of $|\sigma|$ are shown as Fig. 10.

We note that when $|t|$ is small, the higher value of $|\sigma|$ still corresponds to the higher value of $|\omega|$; therefore, the canonical Hilbert transform still works as the edge detection, but when $|t|$ is large, the higher value of $|\sigma|$ will not correspond to the higher value of $|\omega|$. Therefore, when $|t|$ is large, the canonical Hilbert transform will no longer act as the highpass filter and cannot be used for the edge detection, so the canonical Hilbert transform can be used as the localized edge detection. If the edges are in certain region, then the edges can still be detected, but if the edges are out of the region, then they will not be detected. We can use the value of $b / a$ to control the width of the region.

We can also use the fractional convolution for the application of beam shaping [28]. Beam shaping is useful for optical beam synthesis. If the input is $x(t)$, then we try to design a beam shaping filter $h(t)$ to obtain the desired output $y(t)$

$$
y(t)=\left|O_{F}^{\alpha}(x(u) \cdot h(u))\right|
$$

We can also analyze the beam shaping operation as above by the WDF. If

$$
X(t)=O_{F}^{\alpha}(x(u)), \quad H(t)=O_{F}^{\alpha}(h(u))
$$

then

$$
y(t)=\left|X(t) *_{-\alpha} H(t)\right| .
$$

Then, from (14), and the projection property of the WDF

$$
\begin{aligned}
y^{2}(t)= & \int_{-\infty}^{\infty} \int_{-\infty}^{\infty} \\
& \cdot W_{h}(t \cos \alpha-w \sin \alpha, t \sin \alpha+w \cos \alpha-\rho) \\
& \cdot W_{x}(t \cos \alpha-w \sin \alpha, \rho) d \rho d w .
\end{aligned}
$$

That is, if we know the WDF of $x(t)$ and the parameter $\alpha$ has been determined, then we can try to find the beam shaping function $h(t)$ that has the WDF satisfying (64).

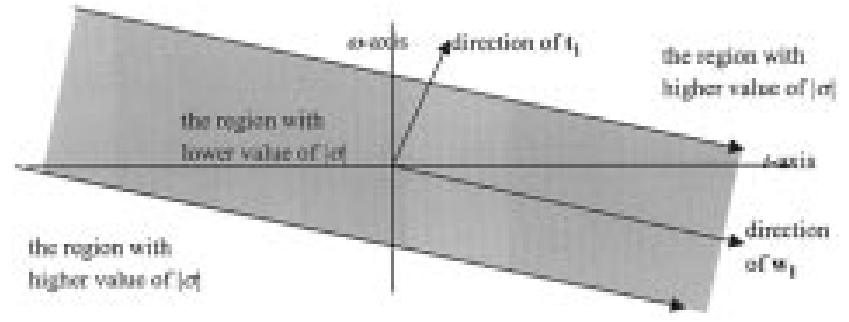

Fig. 10. Regions with high and lower values of $|\sigma|$ for the canonical Hilbert transform.

\section{RELATIONS BETWEEN THE WDF AND FRACTIONAL/CANONICAL CORRELATION}

Suppose that $z(t)$ is the fractional correlation of $x(t)$ and $y(t)$ :

$$
z(t)=O_{c o r r}^{P_{1}, P_{2} \cdot P_{3}}(x(t), y(t))=O_{F}^{P_{3}}\left(X_{P_{1}}(u) \cdot Y_{P_{2}}^{*}(u)\right) .
$$

If

$$
h(u)=X_{P_{1}}(u) \cdot Y_{P_{2}}^{*}(u)
$$

then

$$
\begin{aligned}
& W_{h}(u, v) \\
& =\int_{-\infty}^{\infty} W_{X_{P_{1}}}(u, \rho) \cdot W_{Y_{P_{2}}}(u,-v+\rho) \cdot d \rho \\
& =\int_{-\infty}^{\infty} W_{x}\left(\cos P_{1} \cdot u-\sin P_{1} \cdot \rho, \sin P_{1} \cdot u+\cos P_{1} \cdot \rho\right) \\
& \quad \cdot W_{y}\left(\cos P_{2} \cdot u+\sin P_{2} \cdot(v-\rho)\right. \\
& \left.\quad \sin P_{2} \cdot u-\cos P_{2} \cdot(v-\rho)\right) \cdot d \rho
\end{aligned}
$$

Because $z(t)$ is the FRFT of $h(t)$ with order $-P_{3}$, from relation 1 in Table IV, we obtain

$$
\begin{gathered}
W_{z}(t, w) \\
=\int_{-\infty}^{\infty} W_{x}\left(t \cos P_{1} \cos P_{3}-\omega \cos P_{1} \sin P_{3}-\rho \sin P_{1}\right. \\
\left.\quad t \sin P_{1} \cos P_{3}-\omega \sin P_{1} \sin P_{3}+\rho \cos P_{1}\right) \\
\cdot W_{y}\left(t \cos \left(P_{2}-P_{3}\right)+\omega \sin \left(P_{2}-P_{3}\right)-\rho \sin P_{2}\right. \\
\left.\quad t \sin \left(P_{2}-P_{3}\right)-\omega \cos \left(P_{2}-P_{3}\right)+\rho \cos P 2\right) \cdot d \rho
\end{gathered}
$$

This equation can also be expressed as

$$
\begin{aligned}
W_{z}(t, w)= & \int_{-\infty}^{\infty} W_{x}\left(\sigma \cdot \mathbf{t}_{1}+\rho \cdot \mathbf{w}_{1}\right) \\
& \cdot W_{y}\left(\sigma \cdot \mathbf{t}_{\mathbf{2}}-\mu \cdot \mathbf{w}_{\mathbf{2}}+\rho \cdot \mathbf{w}_{\mathbf{2}}\right) \cdot d \rho
\end{aligned}
$$

where

$$
\begin{array}{rlrl}
\sigma & =t \cos P_{3}-\omega \sin P_{3}, \quad \mu & =t \sin P_{3}+\omega \cos P_{3} \\
\mathbf{t}_{1} & =\left(\cos P_{1}, \sin P_{1}\right), & \mathbf{w}_{1} & =\left(-\sin P_{1}, \cos P_{1}\right) \\
\mathbf{t}_{2} & =\left(\cos P_{2}, \sin P_{2}\right), & \mathbf{w}_{2} & =\left(-\sin P_{2}, \cos P_{2}\right) .
\end{array}
$$

The relation of (69) can be plotted as in Fig. 11. 


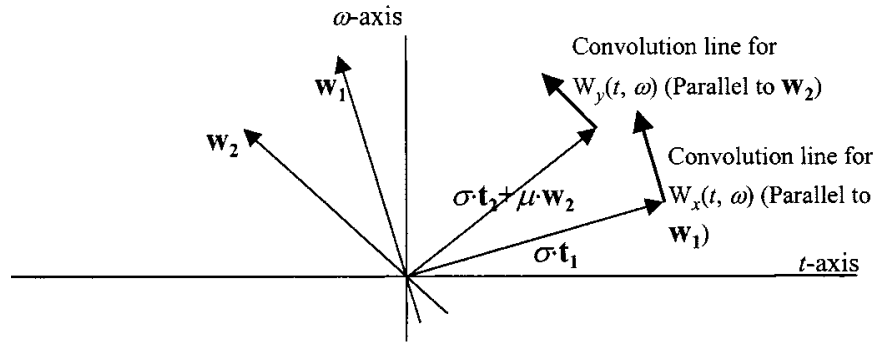

Fig. 11. Relation between WDF and fractional correlation.

We note that the convolution line for $W_{x}(t, \omega)$ is parallel to $\mathrm{w}_{1}=\left(-\sin P_{1}, \cos P_{1}\right)$, and the convolution line for $W_{y}(t, \omega)$ is parallel to $\mathbf{w}_{\mathbf{2}}=\left(-\sin P_{2}, \cos P_{2}\right)$. If $P_{1} \neq P_{2}$, then the direction of convolution line for $W_{x}(t, w)$ will be different from that of the $W_{y}(t, \omega)$. In addition, we note that the role of $P_{3}$ is of less importance because it does not affect the direction of convolution lines. Thus, we usually set $P_{1}=P_{2}$ to make the direction of $\mathbf{w}_{1}$ and $\mathbf{w}_{\mathbf{2}}$ to be the same and set $P_{3}$ as $-\pi / 2$ (i.e., the IFT). If we set $P_{1}=P_{2}$ and $P_{3}=-\pi / 2$, then (69) becomes

$$
\begin{aligned}
W_{z}(t, w)= & \int_{-\infty}^{\infty} W_{x}\left(w \cdot \mathbf{t}_{1}+\rho \cdot \mathbf{w}_{\mathbf{1}}\right) \\
& \cdot W_{y}\left(w \cdot \mathbf{t}_{1}+t \cdot \mathbf{w}_{1}+\rho \cdot \mathbf{w}_{1}\right) \cdot d \rho
\end{aligned}
$$

where

$$
\mathbf{t}_{1}=\left(\cos P_{1}, \sin P_{1}\right), \quad \mathbf{w}_{1}=\left(-\sin P_{1}, \cos P_{1}\right) .
$$

Then, the directions of convolution for $W_{x}(t, \omega), W_{y}(t, \omega)$ in Fig. 11 will be the same.

Similarly, for the canonical correlation defined in Table III, if $z(t)$ is the canonical correlation of $x(t), y(t)$, then their WDFs will have the relation

$$
\begin{aligned}
& W_{z}(t, w) \\
& =\int_{-\infty}^{\infty} W_{x}\left(t \cdot d_{1} d_{3}-\omega \cdot d_{1} b_{3}-\rho \cdot b_{1}\right. \\
& \left.\quad-t \cdot c_{1} d_{3}+\omega \cdot c_{1} b_{3}+\rho \cdot a_{1}\right) \\
& \quad \cdot W_{y}\left(\left(d_{2} d_{3}-b_{2} c_{3}\right) \cdot t+\left(b_{2} a_{3}-d_{2} b_{3}\right) \cdot \omega-\rho \cdot b_{2}\right. \\
& \quad\left(a_{2} c_{3}-c_{2} d_{3}\right) \cdot t+\left(c_{2} b_{3}-a_{2} a_{3}\right) \\
& \left.\quad \cdot \omega+\rho \cdot a_{2}\right) \cdot d \rho .
\end{aligned}
$$

We can also express the above equation as (69), but in this case

$$
\begin{aligned}
\sigma & =t \cdot d_{3}-\omega \cdot b_{3}, & \mu & =-t \cdot c_{3}+\omega \cdot a_{3} \\
\mathbf{t}_{1} & =\left(d_{1},-c_{1}\right), & \mathbf{w}_{1} & =\left(-b_{1}, a_{1}\right) \\
\mathbf{t}_{2} & =\left(d_{2},-c_{2}\right), & \mathbf{w}_{\mathbf{2}} & =\left(-b_{2}, a_{2}\right)
\end{aligned}
$$

and the direction of convolution for the WDF can also be represented as Fig. 11. Similarly, it is convenient to set $\left\{a_{3}, b_{3}, c_{3}, d_{3}\right\}$ as $\{0,-1,1,0\}$, and set $\left\{a_{2}, b_{2}, c_{2}, d_{2}\right\}=\left\{a_{1}, b_{1}, c_{1}, d_{1}\right\}$; then, (73) becomes

$$
\begin{aligned}
W_{z}(t, w)= & \int_{-\infty}^{\infty} W_{x}\left(w d_{1}-\rho b_{1},-w c_{1}+\rho a_{1}\right) \\
& \cdot W_{y}\left(-b_{1} t+d_{1} w-\rho b_{1}, a_{1} t-c_{1} w+\rho a_{1}\right) \cdot d \rho .
\end{aligned}
$$

It is well known that the fractional correlation can be used for space-variant pattern recognition. For this application, we can set $x(t)$ as the reference pattern and $y(t)$ as the input pattern and then calculate the output of the fractional/canonical correlation $z(t)$. Then, if

$$
\operatorname{Max}\left(|z(t)|^{2}\right)>T, \quad \text { where } T \text { is some threshold }
$$

then we can conclude that $y(t)$ is the shifted version of $x(t)$, and the amount of displacement is within a certain region. We will use the relations between the WDF and the fractional/canonical correlation to illustrate why we can use the fractional/canonical correlations for pattern recognition and how to use them efficiently.

Suppose $x(t)$ is a reference pattern and that $y(t)$ is the input object. If $y(t)$ is the shifted version of $x(t)$

$$
y(t)=x^{*}\left(t-t_{0}\right)
$$

then $W_{y}(t, \omega)=W_{x}\left(t-t_{0}, \omega\right)$, and (71) becomes

$$
\begin{aligned}
W_{z}(t, \omega)= & \int_{-\infty}^{\infty} W_{x}\left(\omega \cdot \mathbf{t}_{1}+\rho \cdot \mathbf{w}_{1}\right) \\
& \cdot W_{x}\left(\omega \cdot \mathbf{t}_{1}+(t+\rho) \cdot \mathbf{w}_{1}-t_{0} \cdot \mathbf{t}\right) \cdot d \rho
\end{aligned}
$$

where $\mathbf{t}=(1,0)$. Then, from the projection property of the WDF

$$
\begin{aligned}
|z(t)|^{2}= & \int_{-\infty}^{\infty} \int_{-\infty}^{\infty} W_{x}\left(\omega \cdot \mathbf{t}_{1}+\rho \cdot \mathbf{w}_{1}\right) \\
& \cdot W_{x}\left(\omega \cdot \mathbf{t}_{1}+(t+\rho) \cdot \mathbf{w}_{1}-t_{0} \cdot \mathbf{t}\right) \cdot d \rho \cdot d w \\
= & \int_{-\infty}^{\infty} \int_{-\infty}^{\infty} W_{x}(u, v) \\
& \cdot W_{x}\left(u-t \sin P_{1}-t_{0}, v+t \cos P_{1}\right) \cdot d u \cdot d v .
\end{aligned}
$$

That is, the value of $|z(t)|^{2}$ equals the inner product of $W_{x}(u, v)$, and $W_{x}\left(u-t \cdot \sin P_{1}-t_{0}, v+t \cdot \cos P_{1}\right)$. The vector $t \cdot \mathbf{w}_{1}-t_{0} \cdot \mathbf{t}=\left(-t \cdot \sin P_{1}-t_{0}, t \cdot \cos P_{1}\right)$ is the difference between the locations of $W_{x}(u, v)$ and $W_{x}\left(u-t \cdot \sin P_{1}-t_{0}, v+t \cdot \cos P_{1}\right)$. Usually, if $-t \cdot \sin P_{1}-t_{0}=0$, i.e., the difference of the first coordinate is 0 , then the inner product will have the maximum value. Therefore

$$
\begin{aligned}
\operatorname{Max} & \left(|z(t)|^{2}\right) \\
& =\left|z\left(-t_{0} / \sin P_{1}\right)\right|^{2} \\
& =\int_{-\infty}^{\infty} \int_{-\infty}^{\infty} W_{x}(u, v) W_{x}\left(u, v-t_{0} \cot P_{1}\right) \cdot d u d v
\end{aligned}
$$

The maximum of $|z(t)|^{2}$ is the inner product of $W_{x}(u, v)$ and $W_{x}\left(u, v+t_{0} \cot P_{1}\right)$. If $t_{0} \cot P_{1}$ (the difference of the second coordinate) is small, then $\operatorname{Max}\left(|z(t)|^{2}\right)$ is sufficiently large to satisfy the (76), but if $t_{0} \cot P_{1}$ is too large, then $\operatorname{Max}\left(|z(t)|^{2}\right)$ will be distorted, and (76) will no longer be satisfied. That is, even if the input pattern is the same as the reference, but the displacement $t_{0}$ is too large, then (76) is still not satisfied. Therefore, the object can be identified only when it is the same as the reference and the amount of the displacement is not too large. 


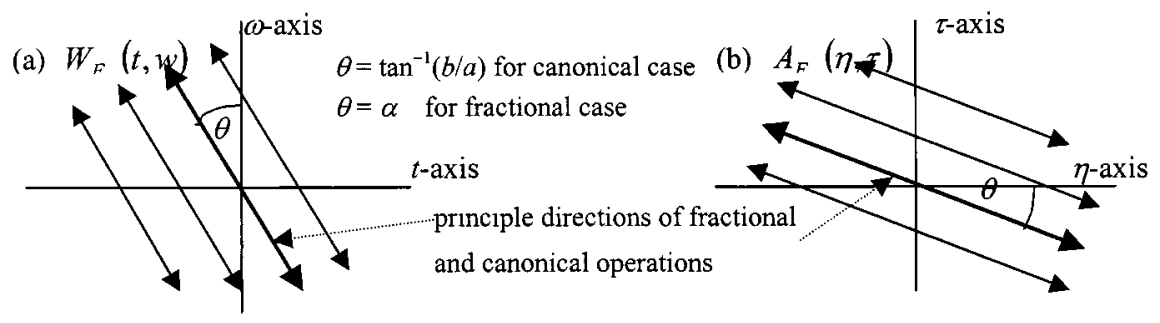

Fig. 12. Principle directions of fractional/canonical operations for (a) WDF and (b) AF.

TABLE V

SOME RELATIONS BETWEEN THE WDF AND THE LCT

\begin{tabular}{l|l}
\hline (1) Recovery property & $\int_{-\infty}^{\infty} W_{f}\left(\frac{d u}{2}-b v,-\frac{c u}{2}+a v\right) \cdot e^{j u v} \cdot d v=F_{(a, b, c, d)}(u) \cdot F_{(a, b, c, d)}^{*}(0)$ \\
\hline $\begin{array}{c}\text { (2) Canonical spectrum } \\
\text { correlation function }\end{array}$ & $\int_{-\infty}^{\infty} W_{f}(d u-b v,-c u+a v) \cdot e^{j v \tau} d v=F_{(a, b, c, d)}\left(u+\frac{\tau}{2}\right) \cdot F_{(a, b, c, d)}^{*}\left(u-\frac{\tau}{2}\right)$ \\
\hline $\begin{array}{l}\text { (3) Mean condition } \\
\text { property }\end{array}$ & If $F_{(a, b, c, d)}(u)=\left|F_{(a, b, c, d)}(u)\right| \cdot e^{j \varphi(u)}$, then \\
& $\varphi^{\prime}(u)=\mid F_{(a, b, c, d)}(u)^{-2} \cdot \int_{-\infty}^{\infty} v \cdot W_{f}(d u-b v,-c u+a v) \cdot d v$ \\
\hline $\begin{array}{l}\text { (4) Mean time-frequency } \\
\text { of WDF }\end{array}$ & $F_{(a, b, c, d)}(u)=\left|F_{(a, b, c, d)}(u)\right| \cdot e^{j \varphi(u)}$, then \\
\hline (5) Moment properties & $\int_{-\infty}^{\infty} \mathcal{L}_{-\infty}^{\infty}(c u+d v) W_{f}(u, v) \cdot d u d v=\int_{-\infty}^{\infty}\left|F_{(a, b, c, d)}(u)\right|^{2} \varphi^{\prime}(u) \cdot d u$ \\
\hline
\end{tabular}

We note that the value of $P_{1}$ can control the range that the object can be detected. If $\cot P_{1}$ is large, then since $t_{0} \cot P_{1}$ grows fast with $t_{0}$, the range that the object can be detected is very narrow. If $\cot P_{1}$ is smaller, then the range will be wider. When $P_{1}=\pi / 2$, as the conventional space-invariant case, then $t_{0} \cot P_{1}$ will always be 0 , and no matter how large the amount of the displacement is, we can always detected the object.

We can also use the generalization of the fractional correlation (the canonical correlation) for space-variant pattern recognition. In this case, (79) becomes

$$
\begin{aligned}
|z(t)|^{2}= & \int_{-\infty}^{\infty} \int_{-\infty}^{\infty} W_{x}(u, v) \\
& \cdot W_{x}\left(u-t b_{1}-t_{0}, v+t a_{1}\right) \cdot d u d v
\end{aligned}
$$

and the difference between the locations of $W_{x}(u, v)$ and $W_{x}\left(u-t \cdot b_{1}-t_{0}, v+t \cdot a_{1}\right)$ is $\left(-t \cdot b_{1}-t_{0}, t \cdot a_{1}\right)$. The maximum of $|z(t)|^{2}$ is at the location where the difference of the first coordinate is 0 , i.e., $-t \cdot b_{1}-t_{0}=0$. Therefore

$$
\begin{aligned}
\operatorname{Max} & \left(|z(t)|^{2}\right) \\
& =\left|z\left(-t_{0} / b_{1}\right)\right|^{2} \\
& =\int_{-\infty}^{\infty} \int_{-\infty}^{\infty} W_{x}(u, v) W_{x}\left(u, v-t_{0} a_{1} / b_{1}\right) \cdot d u d v .
\end{aligned}
$$

The maximum of $|z(t)|^{2}$ will satisfy (76) if $t_{0} a_{1} / b_{1}$ is not too large, so the value of $a_{1} / b_{1}$ can control the width of the range that the object can be detected, and this has the same role as $\cot P_{1}$ for the fractional correlation. We can fix $a_{1}$ as 1 and vary the value of $b_{1}$ to control the width of the range that the object can be detected.

\section{More Properties between the Wigner Distribution FUNCTION AND THE FRACTIONAL/CANONICAL FOURIER TRANSFORM}

Now, we discuss some relations between the WDF and FRFT/LCT, except for the previous relations discussed in Sections III and IV. We list them without proof.

From Properties 1-5 in Table V, we find for the LCT that the directions of integration all intersect the $\omega$-axis with the angle of $\tan ^{-1}(b / a)$ in the counterclockwise direction, as is the case of canonical convolution. Therefore, we can view the vector

$$
\mathbf{w}_{1}=(-b, a)
$$

as the principle direction of the canonical operations on the WDF. For the case of fractional operations, this direction becomes $\mathbf{w}_{1}=(-\sin \alpha, \cos \alpha)$. We draw the principle directions of fractional/canonical operations for the WDF in Fig. 12(a).

The properties listed in Table $\mathrm{V}$ have some applications. From the recovery property, if we know the WDF of $f(t)$ and $F_{(a, b, c, d)}^{*}(u)$ [which is the conjugation of the dc value of the LCT of $f(t)$ ], then we use the recovery property to calculate the LCT of $f(t)$, i.e., $F_{(a, b, c, d)}(u)$.

Property 2 in Table V implies a special method to calculate the WDF. Usually, we calculate the WDF of $f(t)$ from (1). We first calculate $f(t+\tau / 2) \cdot f^{*}(t-\tau / 2)$ for different values of $t$. Then, for each $t$, we calculate the Fourier transform of $f(t+$ $\tau / 2) \cdot f^{*}(t-\tau / 2)$ and then obtain the WDF. From Property 2 

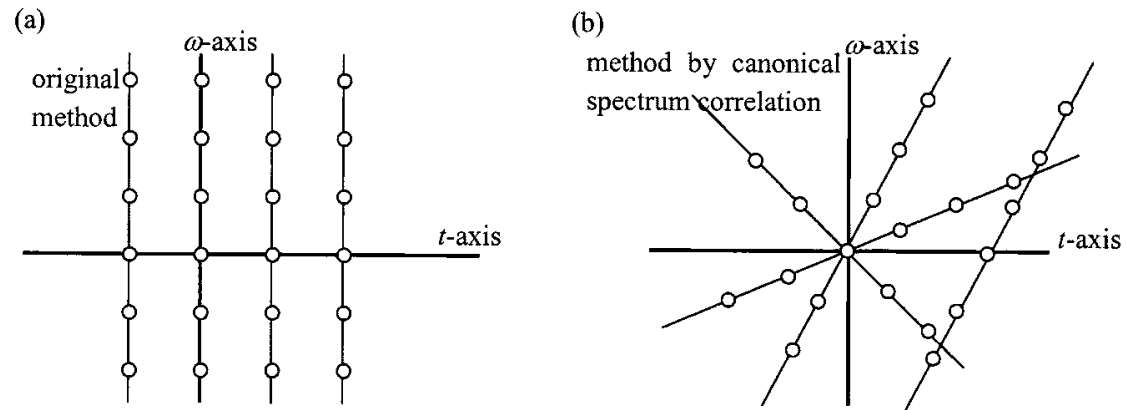

Fig. 13. Location of output sampling points (a) when we use (1) and (b) when we use the canonical spectrum correlation function to calculate the WDF.

in Table V, we can use another method to calculate the WDF. Because

$$
\begin{aligned}
& \frac{1}{2 \pi} \int_{-\infty}^{\infty} F_{(a, b, c, d)}\left(u+\frac{\tau}{2}\right) \cdot F_{(a, b, c, d)}^{*}\left(u-\frac{\tau}{2}\right) \cdot e^{-j v \tau} \cdot d v \\
& \quad=W_{f}(d u-b v,-c u+a v)
\end{aligned}
$$

we can first calculate the LCT of $f(t)$, then calculate the canonical spectrum correlation function $F_{(a, b, c, d)}(u+\tau / 2) \cdot F_{(a, b, c, d)}^{*}(u-\tau / 2)$ for different values of $\{a, b, c, d\}$ and $u$, and then use the Fourier transform to calculate the WDF. The most important advantage of this method is that it is more flexible when controlling the locations of the output sampling points than the original method. For the original method, the output sampling points are at the locations as shown in Fig. 13(a).

$$
\left(t_{k}, n \Delta_{w}\right) \quad n=\ldots,-1,0,1,2,3, \ldots
$$

We can vary the value of $t_{k}$ to control the locations of output sampling points, and for the method described above, the output sampling points are at the locations in Fig. 13(b)

$$
\begin{gathered}
\left(d \cdot u_{k}-b \cdot n \Delta_{v},-a \cdot u_{k}+a \cdot n \Delta_{v}\right) \\
N=\ldots,-1,0,1,2,3, \ldots
\end{gathered}
$$

and we can vary the values of $a, b, c, d$, and $u_{k}$ to control the locations of output sampling points.

Properties (3) and (4) are useful to compute the central time, central frequency problems for the matching pursuit [33]. They are also useful to deal with the group delay problems.

From Property (5), we can obtain the moments of $\left|F_{(a, b, c, d)}(u)\right|^{2}$ from the WDF of $f(t)$. Besides, comparing it with the (4) in Table I, we find that the zeroth moment (i.e., $n=0)$ of $\left|F_{(a, b, c, d)}(u)\right|^{2}$ is all the same as the zeroth moment of $|f(t)|^{2}$ and $|F(\omega)|^{2}$, no matter what the values of $a$, $b, c, d$ are, and the first moment (i.e., $n=1)$ of $\left|F_{(a, b, c, d)}(u)\right|^{2}$ can be expressed as the summation of the first moments of $|f(t)|^{2}$ and $|F(\omega)|^{2}$

$$
\begin{aligned}
& \int_{-\infty}^{\infty} u\left|F_{(a, b, c, d)}(u)\right|^{2} d u \\
& \quad=a \int_{-\infty}^{\infty} t|f(t)|^{2} d t+b \int_{-\infty}^{\infty} \omega|F(\omega)|^{2} d w
\end{aligned}
$$

\section{RELATIONS BETWEEN THE FRACTIONAL OPERATIONS AND THE AMBIGUity FunCTION, SignAL/SPECTRUM CORRELATION FUNCTIONS \\ A. Relations between Ambiguity Function (AF) and FRFT, LCT, and Fractional/Canonical Operations}

First, we will discuss the direct relation between the AF and the LCT. If

$$
\begin{aligned}
& A_{f}(\eta, \tau) \\
& =1 / 2 \pi \cdot \int_{-\infty}^{\infty} f(t+\tau / 2) \cdot f^{*}(t-\tau / 2) \\
& \quad \cdot e^{-j t \eta} \cdot d t \\
& A_{F_{(a, b, c, d)}}(\eta, \tau) \\
& =1 / 2 \pi \cdot \int_{-\infty}^{\infty} F_{(a, b, c, d)}(u+\tau / 2) \\
& \quad \cdot \frac{F_{(a, b, c, d)}(u-\tau / 2)}{F} \cdot e^{-j u \eta} \cdot d u
\end{aligned}
$$

then we can obtain the relation between $A_{F_{(a, b, c, d)}}(\eta, \tau)$ $A_{f}(\eta, \tau)$ and

$$
\begin{aligned}
A_{F_{(a, b, c, d)}}(\eta, \tau) & \mathrm{FT}_{u \rightarrow \eta}\left(\operatorname{IFT}_{v \rightarrow \tau}\left(W_{F_{(a, b, c, d)}}(u, v)\right)\right) \\
= & \mathrm{FT}_{u \rightarrow \eta}\left(\operatorname{IFT}_{v \rightarrow \tau}\left(W_{f}(d u-b v,-c u+a v)\right)\right) \\
= & (2 \pi)^{-1} \int_{-\infty}^{\infty} \int_{-\infty}^{\infty} e^{-j u \eta} e^{j v \tau} W_{f}(d u-b v,-c u+a v) \\
& \cdot d u d v \\
= & (2 \pi)^{-1} \int_{-\infty}^{\infty} \int_{-\infty}^{\infty} e^{-j \tilde{u}(a \eta-c \tau)} e^{j \tilde{v}(-b \eta+d \tau)} W_{f}(\tilde{u}, \tilde{v}) \\
& \cdot d \tilde{u} d \tilde{v} \\
= & A_{f}(a \eta-c \tau,-b \eta+d \tau) .
\end{aligned}
$$

Comparing this equation with Property 2 in Table IV, we find that the $\eta$-axis of the AF has as similar role to the $\omega$-axis of the $\mathrm{WDF}$, and the $\tau$-axis of the AF has a similar role to the $t$-axis of the WDF.

For the case of FRFT

$$
A_{F_{\alpha}}(\eta, \tau)=A_{f}(\cos \alpha \cdot \eta+\sin \alpha \cdot \tau,-\sin \alpha \cdot \eta+\cos \alpha \cdot \tau) .
$$

We find, as the case of WDF, that when we do FRFT for a function, its AF will also rotate by the angle of $\alpha$, but for the case of 
TABLE VI

SOME RELATIONS BETWEEN THE AF AND THE FRFT/LCT

\begin{tabular}{l|l}
\hline $\begin{array}{l}\text { (1) Projection property } \\
\text { (original) }\end{array}$ & $\int_{-\infty}^{\infty} A_{f}(\eta, \tau) \cdot d \eta=f(\tau / 2) \cdot f^{*}(-\tau / 2)$, \\
\hline $\begin{array}{l}\text { (2) Projection property } \\
\text { (for FRFT / LCT) }\end{array}$ & $\int_{-\infty}^{\infty} A_{f}(\eta, \tau) \cdot d \tau=F(\eta / 2) \cdot F^{*}(-\eta / 2)$. \\
\hline $\begin{array}{l}\text { (3) Recovery property } \\
\text { (original) }\end{array}$ & $\int_{-\infty}^{\infty} A_{f}(\cos \alpha \cdot \eta+\sin \alpha \cdot \tau,-\sin \alpha \cdot \eta+\cos \alpha \cdot \tau) \cdot d \eta=F_{\alpha}\left(\frac{\tau}{2}\right) \cdot F_{\alpha}^{*}\left(-\frac{\tau}{2}\right)$, \\
\hline $\begin{array}{l}\text { (4) Recovery property } \\
\text { (for FRFT / LCT) }\end{array}$ & $\int_{-\infty}^{\infty} A_{f}(\eta, t) \cdot e^{j \cdot t \eta / 2} \cdot d \eta=f(t) \cdot f^{*}(0)$, \\
\hline $\begin{array}{l}\text { (5) Spectrum correlation } \\
\text { function }\end{array}$ & $\int_{-\infty}^{\infty} A_{f}(\eta \eta, \tau) \cdot e^{-j \cdot w \tau / 2} \cdot d \tau=F(\omega) \cdot F^{*}(0)$. \\
(for FRFT/LCT) & $\int_{-\infty} A_{f}(\eta \cos \alpha+\tau \sin \alpha,-\eta \sin \alpha+\tau \cos \alpha) e^{j u \eta} d \eta=F_{\alpha}\left(u+\frac{\tau}{2}\right) F_{\alpha}^{*}\left(u-\frac{\tau}{2}\right)$, \\
\hline
\end{tabular}

(6) Modulation and time- If $y(t)=e^{j n t} \cdot x(t-m)$, then

\begin{tabular}{l|l} 
shifting & $A_{y}(t, \tau)=e^{j \cdot(n \tau-m \eta)} A_{x}(t, \tau)$.
\end{tabular}

TABLE VII

Relations betweEn the AF and the Fractional/CANonical OPERATIONS

\begin{tabular}{l|l}
\hline (1) Multiplication & If $z(t)=x(t) \cdot y(t)$, then $A_{z}(\eta, \tau)=\int_{-\infty}^{\infty} A_{x}(v, \tau) \cdot A_{y}(\eta-v, \tau) \cdot d v$. \\
\hline (2) Convolution & If $z(t)=x(t) * y(t)$, then $A_{z}(\eta, \tau)=2 \pi \int_{-\infty}^{\infty} A_{x}(\eta, \rho) A_{y}(\eta, \tau-\rho) \cdot d \rho$. \\
\hline (3) Correlation & If $z(t)=x(t) \otimes y(t)=x(t) * y^{*}(-t)$, then \\
& $A_{z}(\eta, \tau)=2 \pi \cdot \int_{-\infty}^{\infty} A_{x}(\eta, \rho) \cdot A_{y}(-\eta, \tau-\rho) \cdot d \rho$. \\
\hline $\begin{array}{l}\text { (4) Hilbert } \\
\text { transform }\end{array}$ & If $y(t)$ is the original Hilbert transform of $x(t)$, then \\
& $A_{y}(\eta, \tau)=-\int_{-\infty}^{\infty} A_{x}(\eta, \tau-\rho) \cdot 2 \sin (2|\eta| \rho) \cdot \frac{d \rho}{\pi \rho}+A_{x}(\eta, \tau)$. \\
\hline
\end{tabular}

the WDF, the direction of rotation is clockwise, and the direction of rotation for the case of $\mathrm{AF}$ is counterclockwise.

From the relation between the AF and the LCT, we can also find the relations between the $\mathrm{AF}$ and some important operations. For the Fresnel transform and scaling operation

$$
\begin{array}{ll}
\text { Fresnel transform: } & g_{\sigma}(t)=(2 \pi|\sigma|)^{1 / 2} \cdot f(t) * e^{j t^{2} / 2 \sigma} \\
\text { Scaling operation: } & h_{\rho}(t)=|\rho|^{-1 / 2} \cdot f(t / \rho)
\end{array}
$$

they correspond to the LCT with parameters $\{1, \sigma, 0,1\}$ and $\left\{\rho, 0,0, \rho^{-1}\right\}$, so from (89)

$$
\begin{aligned}
& A_{g_{\sigma}}(\eta, \tau)=A_{f}(\eta,-\sigma \eta+\tau) \\
& A_{h_{\rho}}(\eta, \tau)=A_{f}(\rho \eta, \tau / \rho) .
\end{aligned}
$$

From (89), (90), and the relation between the WDF and AF, and together with the properties of the WDF, we can derive some relations with the AF and the FRFT/LCT. We present some of them in Table VI. From Properties 2, 4, and 5, we find for the LCT that the integration lines all intersect the $t$-axis with the angle of $\tan ^{-1}(b / a)$ in the clockwise direction. For the FRFT, this angle becomes $\alpha$. Thus, we can view the principle direction for the canonical operation on the ambiguity function as

$$
\vec{e}=(a,-b) \text {. }
$$

For the fractional operations, the principle direction becomes $(\cos \alpha,-\sin \alpha)$. We draw the principle direction of the fractional and canonical operations on the AF in Fig. 12(b) and compare it with the case of WDF, which is plotted in Fig. 12(b).

Then, we discuss the relations between the AF and some other fractional/canonical operations. We list them in Table VIII. We can compare them with Table VII, which are the relations between the AF and some conventional operations. 
TABLE VIII

RELATIONS BETWEEN THE AF AND THE FRACTIONAL/CANONICAL OPERATIONS

\begin{tabular}{|c|c|}
\hline $\begin{array}{l}\text { (1) Canonical } \\
\text { convolution }\end{array}$ & $\begin{array}{l}\text { If } z(t)=x(t) *_{(a, b, c, d)} y(t) \text {, then } \\
\quad A_{z}(\eta, \tau)=\int_{-\infty}^{\infty} A_{x}(a v-c(b \eta+a \tau),-b v+d(b \eta+a \tau)) \\
\qquad \cdot A_{y}(-a v+\eta, b v+\tau) \cdot d v \\
\text { The fractional convolution is the special case of it that }\{a, b, c, d\}=\{\cos \alpha, \\
\sin \alpha,-\sin \alpha, \cos \alpha\} .\end{array}$ \\
\hline & $\begin{array}{l}\text { If } z(t)=O_{F_{\left(a_{3}, b_{3}, c_{3}, d_{3}\right)}}\left(X_{\left(a_{1}, b_{1}, c_{1}, d_{1}\right)}(u) \cdot Y_{\left(a_{2}, b_{2}, c_{2}, d_{2}\right)}^{*}(u)\right), \text { then } \\
\begin{array}{l}A_{z}(\eta, \tau)=\int_{-\infty}^{\infty} A_{x}\left(c_{1} b_{3} \cdot \eta-c_{1} d_{3} \cdot \tau+a_{1} \cdot v,-d_{1} b_{3} \cdot \eta+d_{1} d_{3} \cdot \tau+a_{1} \cdot v\right) . \\
A_{y}\left(\left(c_{2} b_{3}-a_{2} a_{3}\right) \eta+\left(a_{2} c_{3}-c_{2} d_{3}\right) \tau+a_{2} \cdot v,\right. \\
\left.\quad\left(b_{2} a_{3}-d_{2} b_{3}\right) \eta+\left(d_{2} d_{3}-b_{2} c_{3}\right) \tau-b_{2} \cdot v\right) \cdot d v\end{array} \\
\text { The fractional correlation is the special case of it that }\{a, b, c, d\}=\{\cos \alpha, \\
\sin \alpha,-\sin \alpha, \cos \alpha\} .\end{array}$ \\
\hline $\begin{array}{l}\text { (3) Canonical } \\
\text { Hilbert } \\
\text { transform }\end{array}$ & $\begin{array}{l}\text { If } y(t)=O_{H l}^{(a, b, c, d), \phi}(x(t)) \text {, then } \\
\begin{aligned} A_{y}(\eta, \tau)= & \int_{-\infty}^{\infty} A_{x}(a v-c(b \eta+a \tau),-b v+d(b \eta+a \tau)) \cdot \sin (-a v+\eta \cdot(b v+\tau) / 2) \\
& \cdot(\pi(b v+\tau))^{-1} \cdot\left(-1+e^{j 2 \phi} u(-a v+\eta)+e^{-j 2 \phi}(1-u(-a v+\eta))\right) d v \\
& +A_{x}(-a \tau / b-c(b \eta+a \tau), \tau+d(b \eta+a \tau))\end{aligned} \\
\text { Fractional Hilbert transform is the special case that }\{a, b, c, d\}=\{\cos \alpha, \\
\sin \alpha,-\sin \alpha, \cos \alpha\} .\end{array}$ \\
\hline
\end{tabular}

\section{B. Relations between Signal/Spectrum Correlation Function and Fractional/Canonical Fourier Transform}

The signal correlation function [which is defined as (3)] and the spectrum correlation function [which is defined as (4)] also have some relations with the fractional/canonical operations, although these relations are not as simple as those of the WDF and AF.

We first give the definition of canonical signal correlation function as

$$
\begin{gathered}
C_{F_{(a, b, c, d)}}(u, \tau)=(2 \pi)^{-1 / 2} F_{(a, b, c, d)}(u+\tau / 2) \\
\cdot F_{(a, b, c, d)}^{*}(u-\tau / 2)
\end{gathered}
$$

and the canonical spectrum correlation function can be defined as

$$
\begin{aligned}
S_{F_{(a, b, c, d)}}(\eta, v)= & (2 \pi)^{-1 / 2} F_{(c, d,-a,-b)}(v+\eta / 2) \\
& \cdot F_{(c, d,-a,-b)}^{*}(v-\eta / 2) .
\end{aligned}
$$

The fractional signal /spectrum correlation functions are the special cases of (94) and (95) where $\{a, b, c, d\}=$ $\{\cos \alpha, \sin \alpha,-\sin \alpha, \cos \alpha\}$. The relation between the canonical signal correlation function and the canonical spectrum correlation function is

$$
S_{F_{(a, b, c, d)}}(\eta, v)=C_{F_{(c, d,-a,-b)}}(v, \eta) .
$$

1) Relation between the Fractional/Canonical Signal Correlation Function and the FRFT/LCT:

$$
\begin{aligned}
C_{F_{(a, b, c, d)}}(u, \tau)= & \frac{1}{2 \pi|b|} e^{j(d / b) \cdot u \tau} \int_{-\infty}^{\infty} \int_{-\infty}^{\infty} e^{-(j / b)(u \rho+\tau t)} \\
& \cdot e^{j(a / b) \cdot t \rho} \cdot C_{f}(t, \rho) \cdot d t d \rho .
\end{aligned}
$$

In other words

$$
\begin{aligned}
C_{F_{(a, b, c, d)}}(u, \tau) \\
=\exp \left(j \frac{d}{b} \cdot u \tau\right) \\
\quad \cdot O_{S c}^{b}\left(\mathrm{FT}_{t \rightarrow \tau, \rho \rightarrow u}\left(\exp \left(j \frac{a}{b} \cdot t \rho\right) \cdot C_{f}(t, \rho)\right)\right)
\end{aligned}
$$

where the scaling operation $O_{s c}^{b}$ is defined as

$$
O_{S c}^{b}(g(x, y))=|b|^{-1} \cdot g(x / b, y / b)
$$

For the case of FRFT $(\{a, b, c, d\}=\{\cos \alpha, \sin \alpha$, $-\sin \alpha, \cos \alpha\})$

$$
\begin{aligned}
& C_{F_{\alpha}}(u, \tau) \\
& \quad=e^{j \cot \alpha \cdot u \tau} \cdot O_{S c}^{\sin \alpha}\left(\mathrm{FT}_{t \rightarrow \tau, \rho \rightarrow u}\left(e^{j \cot \alpha \cdot t \rho} \cdot C_{f}(t, \rho)\right)\right) .
\end{aligned}
$$

Therefore, we can convert the signal correlation function of $f(t)$ into the signal correlation function of $F_{(a, b, c, d)}(u)$ or $F_{\alpha}(u)$ by two multiplication operations: one 2-D Fourier transform and one scaling operation.

2) Relation between the Fractional/Canonical Spectrum Correlation Function and the FRFT/LCT: If $F_{(a, b, c, d)}(u)$ is the LCT of $f(t)$ and $F_{\alpha}(u)$ is the FRFT of $f(t)$, then their 
spectrum correlation function will have the same relation as the signal correlation function. That is

$$
\begin{aligned}
& S_{F_{(a, b, c, d)}}(u, \tau) \\
& \quad=e^{j(d / b) \cdot u \tau} \cdot O_{S c}^{b}\left(\mathrm{FT}_{t \rightarrow \tau, \rho \rightarrow u}\left(e^{j(a / b) \cdot t \rho} \cdot S_{f}(t, \rho)\right)\right)
\end{aligned}
$$

$$
\begin{aligned}
& S_{F_{\alpha}}(u, \tau) \\
& \quad=e^{j \cot \alpha \cdot u \tau} \cdot O_{S c}^{\sin \alpha}\left(\mathrm{FT}_{t \rightarrow \tau, \rho \rightarrow u}\left(e^{j \cot \alpha \cdot t \rho} \cdot S_{f}(t, \rho)\right)\right) .
\end{aligned}
$$

3) Relation between the Canonical Signal Correlation Function and the Canonical Hilbert Transform: If $y(t)$ is the canonical Hilbert transform of $x(t)$

$$
\begin{aligned}
y(t) & =O_{H l}^{(a, b, c, d), \phi}(x(t)) \\
& =O_{F}^{(d,-b,-c, a)}\left(H_{\phi}(u) \cdot O_{F}^{(a, b, c, d)}(x(t))\right)
\end{aligned}
$$

where $H_{\phi}(u)$ is defined as $(51)$, then

$$
C_{Y_{(a, b, c, d)}}(u, \tau)=C_{X_{(a, b, c, d)}}(u, \tau) \cdot M(u, \tau)
$$

where

$$
\begin{aligned}
& M(u, \tau)=1, \quad \text { when } t>\tau / 2 \text { or } t<-\tau / 2 \\
& M(u, \tau)=e^{j 2 \phi}, \quad \text { when }-\tau / 2<t<\tau / 2 .
\end{aligned}
$$

\section{CONCLUSIONS}

We have used the Wigner distribution function (WDF) and the ambiguity function (AF) to illustrate the effects of the FRFT/LCT, fractional/canonical convolution, correlation, and Hilbert transforms and use them to illustrate the effects of the fractional/canonical operations.

From the relations between WDF/AF and the fractional/canonical operations, it will be easier to see their effects on the signal, as well as some applications and some design rules. For many applications about the FRFT, such as fractional filter design, we require the iterative algorithm to search the parameters. In fact, if we know the WDF or the AF of the signal, it would be easier to use the relations discussed in this paper to choose the parameters we want. For example, in Section III-A, we introduce a method to design the pass-stopband filter in the fractional domain from the WDF; this method is very simple and clear in concept.

\section{REFERENCES}

[1] V. Namias, "The fractional order Fourier transform and its application to quantum mechanics," J. Inst. Math. Appl., vol. 25, pp. 241-265, 1980.

[2] L. B. Almeida, "The fractional Fourier transform and time-frequency representations," IEEE Trans. Signal Processing, vol. 42, pp. 3084-3091, Nov. 1994

[3] H. M. Ozaktas, M. A. Kutay, and D. Mendlovic, "Introduction to the fractional Fourier transform and its applications," in Advances in Imaging and Electron Physics. New York: Academic, 1999, vol. 106, ch. 4 .

[4] N. Wiener, "Hermitian polynomials and Fourier analysis," J. Math. Phys. MIT, vol. 18, pp. 70-73, 1929.

[5] A. I. Zayed, "On the relationship between the Fourier transform and fractional Fourier transform," IEEE Signal Processing Lett., vol. 3, pp. 310-311, Dec. 1996
[6] K. B. Wolf, Integral Transforms in Science and Engineering. New York: Plenum, 1979, ch. 9: Canonical transforms.

[7] M. Moshinsky and C. Quesne, "Linear canonical transformations and their unitary representations," J. Math. Phys., vol. 12, no. 8, pp. 1772-1783, Aug. 1971.

[8] S. Abe and J. T. Sheridan, "Optical operations on wave functions as the Abelian subgroups of the special affine Fourier transformation," Opt. Lett., vol. 19, no. 22, pp. 1801-1803, 1994.

[9] A. Papoulis, Signal Analysis. New York: McGraw-Hill, 1977.

[10] L. M. Bernardo, "ABCD matrix formalism of fractional Fourier optics," Opt. Eng., vol. 35, no. 3, pp. 732-740, Mar. 1996.

[11] E. P. Wigner, "On the quantum correlation for thermodynamic equilibrium," Phys. Rev., vol. 40, pp. 749-759, 1932.

[12] F. Hlawatsch and G. F. Boudreaux-Bartels, "Linear and quadratic time-frequency signal representation," IEEE Signal Processing Mag., pp. 21-67, Apr. 1992.

[13] T. A. C. M. Classen and W. F. G. Mecklenbrauker, "The Wigner distribution-A tool for time-frequency signal analysis; Part I: Continuous time signals," Philips J. Res., vol. 35, pp. 217-250, 1980.

[14] A. W. Lohmann, "Image rotation, Wigner rotation, and the fractional Fourier transform," J. Opt. Soc. Amer. A, vol. 10, no. 10, pp. 2181-2186, Oct. 1993.

[15] H. M. Ozaktas, B. Barshan, D. Mendlovic, and L. Onural, "Convolution, filtering, and multiplexing in fractional Fourier domains and their rotation to chirp and wavelet transform," J. Opt. Soc. Amer. A, vol. 11, no. 2, pp. 547-559, Feb. 1994.

[16] A. W. Lohmann and B. H. Soffer, "Relationship between the RadonWigner and the fractional Fourier transform," J. Opt. Soc. Amer. A, vol. 11, no. 6, pp. 1798-1801, 1994.

[17] L. B. Almeida, "Product and convolution theorems for the fractional Fourier transform," IEEE Signal Processing Lett., vol. 4, pp. 15-17, Jan. 1997.

[18] A. I. Zayed, "A convolution and product theorem for the fractional Fourier transform," IEEE Signal Processing Lett., vol. 5, pp. 101-103, Apr. 1998.

[19] D. Mendlovic, H. M. Ozaktas, and A. W. Lohmann, "Fractional correlation," Appl. Opt., vol. 34, no. 2, pp. 303-309, Jan. 1995.

[20] A. W. Lohmann, Z. Zalevsky, and D. Mendlovic, "Synthesis of pattern recognition filters for fractional Fourier processing," Opt. Commun., vol. 128, pp. 199-204, July 1996.

[21] A. W. Lohmann and D. Mendlovic, "Fractional joint transform correlator," Appl. Opt., vol. 36, no. 29, pp. 7402-7407, Oct. 1997.

[22] A. W. Lohmann, D. Mendlovic, and Z. Zalevsky, "Fractional Hilbert transform," Opt. Lett., vol. 21, no. 4, pp. 281-283, Feb. 1996.

[23] A. I. Zayed, "Hilbert transform associated with the fractional Fourier transform," IEEE Signal Processing Lett., vol. 5, pp. 206-208, Aug. 1998.

[24] A. W. Lohmann, D. Mendlovic, and Z. Zalevsky, "Fractional transformations in optics," in Progress in Optics. New York: Elsevier, 1998, vol. 38 , ch. 4

[25] M. A. Kutay, H. M. Ozaktas, O. Arikan, and L. Onural, "Optimal filters in fractional Fourier domain," IEEE Trans. Signal Processing, vol. 45, pp. 1129-1143, May 1997.

[26] B. Barshan, M. A. Kutay, and H. M. Ozaktas, "Optimal filters with linear canonical transformations," Opt. Commun., vol. 135, pp. 32-36, 1997.

[27] Z. Zalevsky and D. Mendlovic, "Fractional Wiener filter," Appl. Opt., vol. 35, no. 20, pp. 3930-3936, July 1996.

[28] Z. Zalevsky, D. Mendlovic, and R. G. Dorsch, "Gerchberg-Saxton algorithm applied in the fractional Fourier or the Fresnel domain," Opt. Lett., vol. 21, no. 2, pp. 842-844, June 1996.

[29] J. J. Ding, "Derivation and properties of orthogonal transform," M.S. thesis, National Taiwan Univ., Taipei, Taiwan, R.O.C., 1997.

[30] R. N. Bracewell, The Fourier Transform and Its Applications, 3rd ed. New York: McGraw-Hill, 2000.

[31] D. A. Mustard, "The fractional Fourier transform and the Wigner distribution," School Math. Preprint, Univ. New South Wales, Kensington, Australia, 1989.

[32] - "The fractional Fourier transform and the Wigner distribution," $J$. Aust. Math. Soc. B, vol. 38, pp. 209-219, 1996.

[33] S. G. Mallat and Z. Zhang, "Matching pursuits with time-frequency dictionaries," IEEE Trans. Signal Processing, vol. 41, pp. 3397-3415, Dec. 1993.

[34] D. Mendlovic, H. M. Ozaktas, and A. W. Lohmann, "Graded-index fibers, Wigner distribution and the fractional Fourier transform," Appl. Opt., vol. 33, pp. 6188-6193, 1994. 


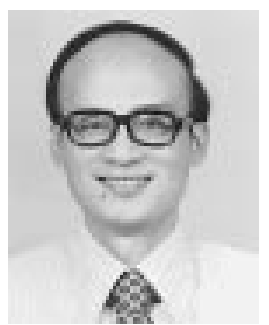

Soo-Chang Pei (F'00) was born in Soo-Auo, Taiwan, R.O.C., in 1949. He received the B.S.E.E. from National Taiwan University (NTU), Taipei, in 1970 and the M.S.E.E. and Ph.D. degrees from the University of California, Santa Barbara (UCSB), in 1972 and 1975, respectively.

He was an Engineering Officer with the Chinese Navy Shipyard from 1970 to 1971. From 1971 to 1975 , he was a Research Assistant at UCSB. He was Professor and Chairman in the Electrical Engineering Department, Tatung Institute of Technology and NTU, from 1981 to 1983 and 1995 to 1998, respectively. Presently, he is a Professor with the Electrical Engineering Department, NTU. His research interests include digital signal processing, image processing, optical information processing, and laser holography.

Dr. Pei received the National Sun Yet-Sen Academic Achievement Award in Engineering in 1984, the Distinguished Research Award from the National Science Council from 1990 to 1998, the Outstanding Electrical Engineering Professor Award from the Chinese Institute of Electrical Engineering in 1998, and the Academic Achievement Award in Engineering from the Ministry of Education in 1998. He was President of the Chinese Image Processing and Pattern Recognition Society in Taiwan from 1996 to 1998 and is a member of Eta Kappa $\mathrm{Nu}$ and the Optical Society of America.

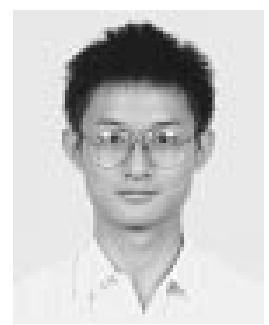

Jian-Jiun Ding was born in 1973 in Pingdong, Taiwan, R.O.C. He received both the B.S. and M.S. degrees in electrical engineering from National Taiwan University (NTU), Taipei, in 1995 and 1997, respectively. He is currently pursuing the Ph.D. degree under the supervision of Prof. S.-C. Pei with the Department of Electrical Engineering at NTU.

His current research areas include the fractional Fourier transform, the linear canonical transform, other fractional transforms, the Hilbert transform, fast algorithms, orthogonal polynomials, integer transforms, the quaternion Fourier transform, pattern recognition, fractals, filter design, etc. 